HOCHSCHULE

MITTWEIDA

UNIVERSITY OF

APPLIED SCIENCES

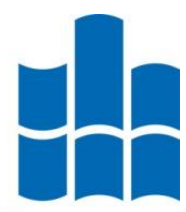

BACHELORARBEIT

Herr

Nic Krumbiegel

Motivation von Mitarbeitern 


\title{
BACHELORARBEIT
}

\section{Motivation von Mitarbeitern}

\author{
Autor: \\ Herr Nic Krumbiegel \\ Studiengang: \\ Business Management
}

Seminargruppe:

BM12wI2-B

Erstprüfer:

Prof. Dr. sc. med. Thomas Müller

Zweitprüfer: Dipl.-Ök. Thomas Franke-Gernhardt

Mittweida, 24.06.2015 


\title{
BACHELOR THESIS
}

\section{Employee Motivation}

\author{
author: \\ Mr. Nic Krumbiegel \\ course of studies: \\ Business Management
}

seminar group:

BM12wl2-B

first examiner:

Prof. Dr. sc. med. Thomas Müller

second examiner: Dipl.-Ök. Thomas Franke-Gernhardt

submission:

Mittweida, 24.06.2015 


\section{Bibliografische Angaben}

Nachname, Vorname:

Krumbiegel, Nic

Thema der Bachelorarbeit:

Motivation von Mitarbeitern

Topic of thesis:

Employee Motivation

61 Seiten, Hochschule Mittweida, University of Applied Sciences,

Fakultät Medien, Bachelorarbeit, 2015

\section{Abstract}

Die Mitarbeiter eines Unternehmens sind dessen wichtigstes Kapital. Umso wichtiger ist es, dass diese motiviert sind. Sollte das der Fall sein, werden sie ihre volle Leistungsbereitschaft zeigen, neue Ideen in das Unternehmen einbringen und dazu beitragen, dass dieses in Zukunft konkurrenzfähig bleibt. Studien zufolge ist es jedoch nur ein kleiner Anteil der Mitarbeiter, die ihrer Arbeit motiviert nachgehen. Die folgende Arbeit geht deshalb der Frage nach, wie Mitarbeiter durch den Arbeitgeber oder Vorgesetzten motiviert werden können, da dies vor allem in deren Interesse sein sollte. Dabei werden die wichtigsten Motivationstheorien erläutert, die häufigsten Fehler in der Motivation von Mitarbeitern aufgezeigt, und anhand eines realen Fallbeispieles, Handlungsempfehlungen für dieses abgeleitet. 


\section{Inhaltsverzeichnis}

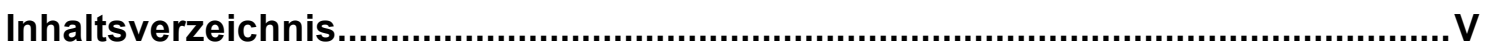

Abbildungsverzeichnis .......................................................................................

Tabellenverzeichnis............................................................................................ VII

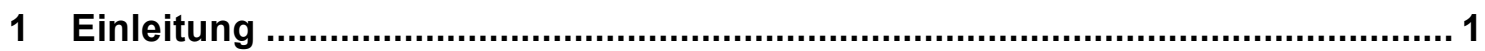

1.1 Heranführung an die Thematik und Begründung der Themenwahl............... 1

1.2 Grundlegende Fragestellung und Abgrenzung des Themas ........................ 3

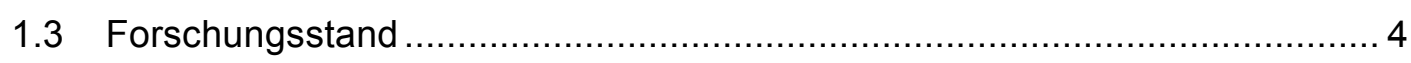

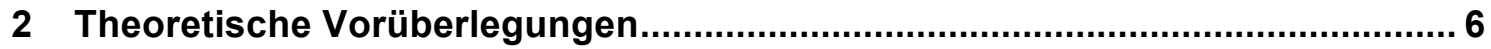

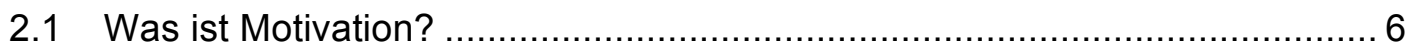

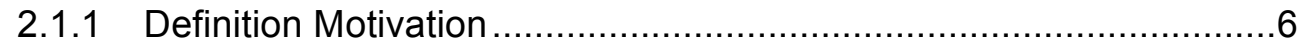

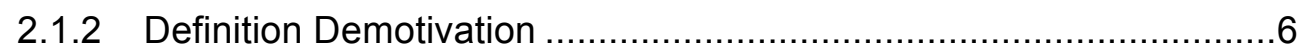

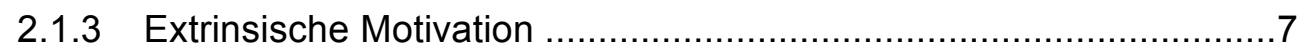

2.1.4 Intrinsische Motivation ..............................................................

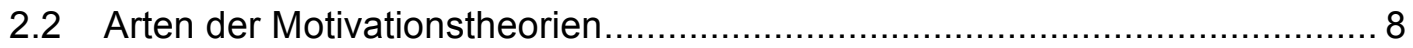

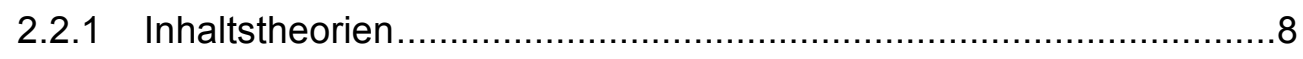

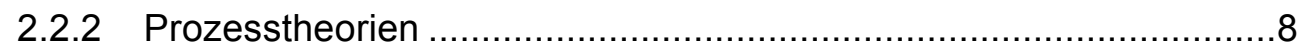

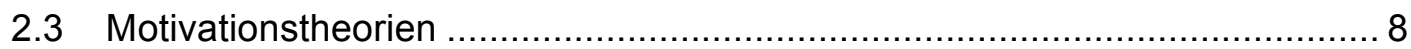

2.3.1 Bedürfnistheorie nach Maslow ..................................................

2.3.2 ERG-Theorie nach Alderfer ................................................. 11

2.3.3 Zwei-Faktoren-Theorie nach Herzberg ......................................12

2.3.4 X-Y-Theorie von McGregor ................................................14

2.3.5 Valenz-Instrumentalitäts-Erwartungstheorie nach Vroom ..............15

2.3.6 Zielsetzungstheorie nach Locke und Latham ...............................17

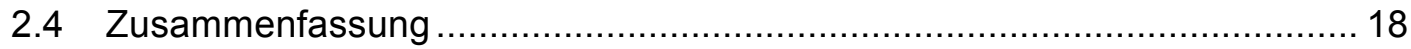

2.4.1 Gemeinsamkeiten der Theorien .............................................18

3 Motivation in der wirtschaftlichen Praxis ......................................................... 20

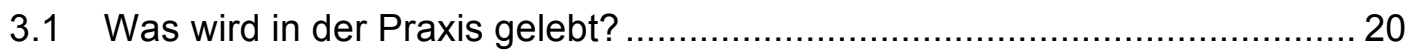

3.2 Folgen fehlender Motivation der Mitarbeiter .............................................. 22

3.3 Wie können Mitarbeiter motiviert werden? ............................................... 23

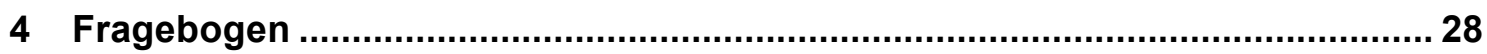

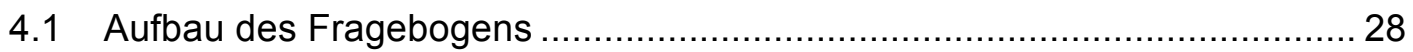

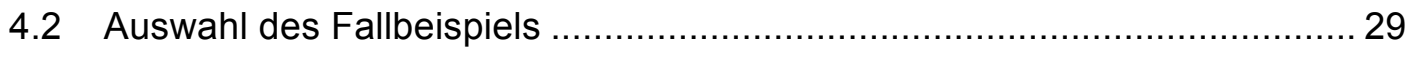




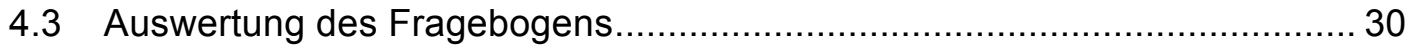

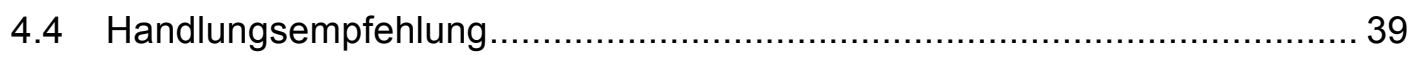

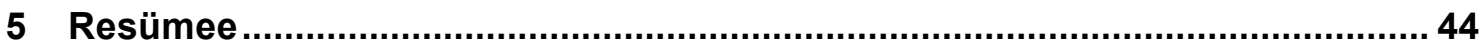

5.1 Zusammenfassung und Reflektion der Ergebnisse............................. 44

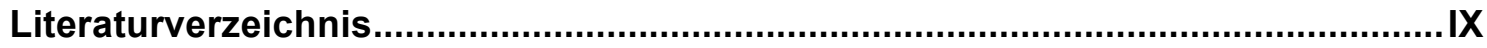

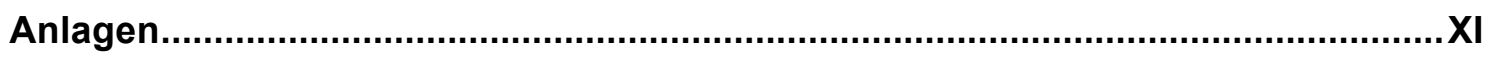

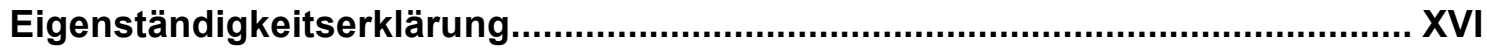




\section{Abbildungsverzeichnis}

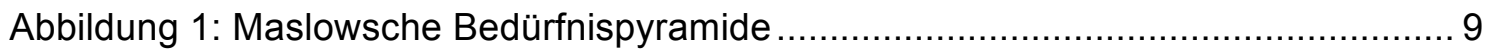

Abbildung 2: ERG-Theorie im Vergleich zur Bedürfnispyramide .............................. 11

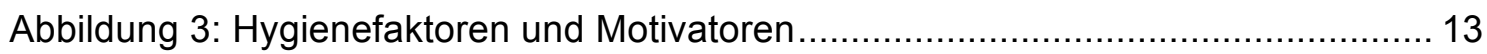

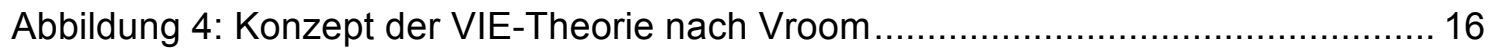

Abbildung 5: Zielsetzungstheorie nach Locke und Latham .................................... 17

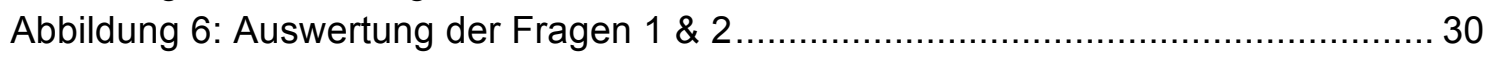

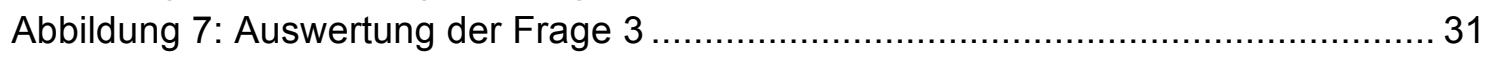

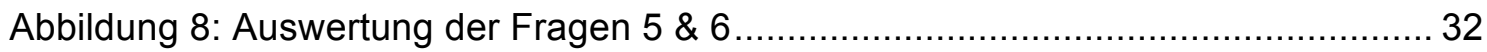

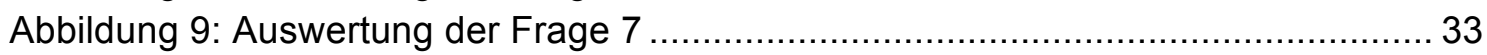

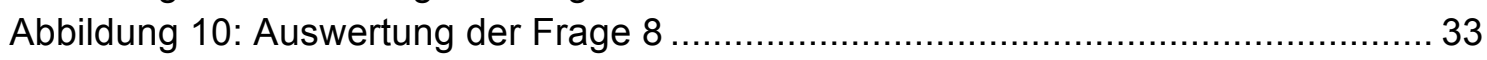

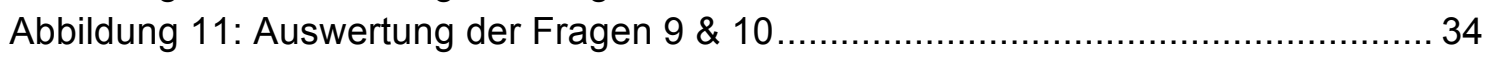

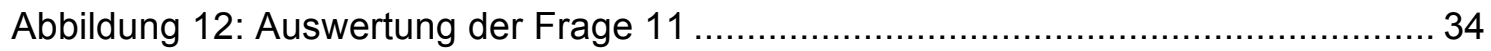

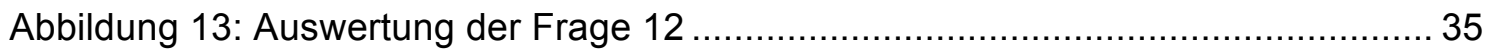

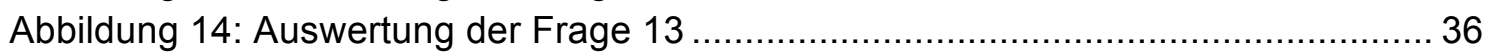

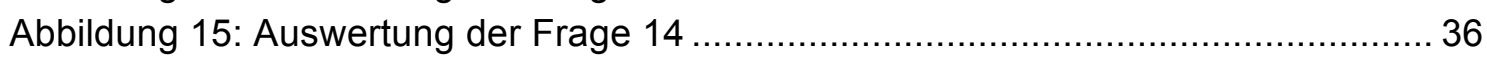

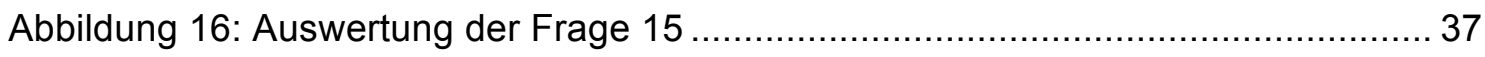

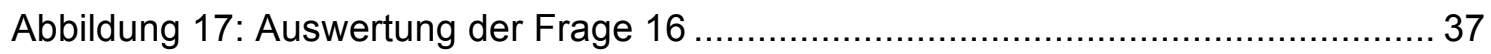

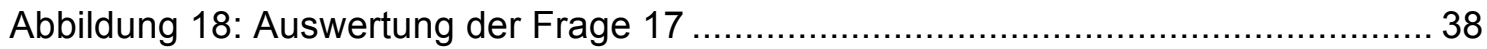

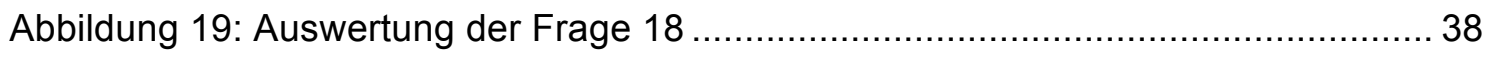

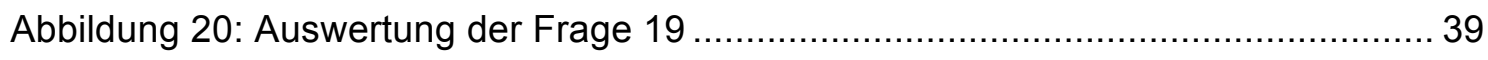




\section{Tabellenverzeichnis}

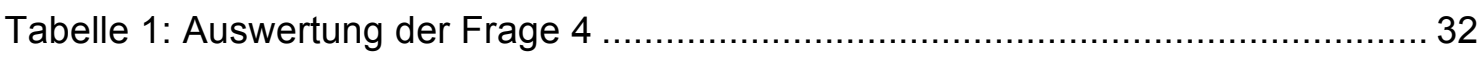




\section{Einleitung}

\subsection{Heranführung an die Thematik und Begründung der Themenwahl}

„Wir wissen zwar, dass ein niedriges Gehalt Menschen demotiviert. D.h. aber im Umkehrschluß nicht, dass sie besonders motiviert sind, wenn sie hochbezahlt sind. "1 Rüdiger Hossiep

Das Herzstück eines jeden Unternehmens sind seine Angestellten. Um aber einen nachhaltigen Unternehmenserfolg gewährleisten zu können, ist es wichtig, dass die Mitarbeiter nicht nur ihrer Regelarbeitszeit nachkommen, sondern auch Motivation und Verbundenheit mit dem Unternehmen zeigen.

Nur allzu oft wird davon ausgegangen, dass mangelnde Motivation mittels monetärer Anreize, beispielsweise Prämien, wieder erweckt werden kann. Doch der Einsatz dieser oder anderer materieller Vergütungen sorgt oft nur für kurze Motivationsschübe, nicht aber für eine längerfristige Zufriedenheit der Angestellten. Mittels Arbeitsnehmerbefragungen fand man heraus, dass der Faktor Bezahlung, bei einer Auflistung von mehreren Motivationsfaktoren, lediglich im Mittelfeld landete und somit nicht der ausschlaggebende Punkt für einen motivierten Mitarbeiter darstellt. ${ }^{2}$

Dies zeigt sich auch anhand einer Studie des renommierten US-amerikanischen Beratungsunternehmens Gallup, welche seit 2001 einmal jährlich für Deutschland erhoben wird. Hierbei werden ca. 2000 Arbeitnehmer/innen ab einem Alter von 18 Jahren, nach einem mehrstufigen Zufallsprinzip befragt. Die Auswertung für das Jahr 2014 ergab, dass zwar 52\% der Befragten die Bezahlung ihrer Arbeit als angemessen empfinden, jedoch lediglich $15 \%$ hochmotiviert sind und eine emotional starke Bindung zu ihrem Unternehmen aufweisen. Der Großteil der Arbeitnehmer/innen (70\%) verrichtet ausschließlich seine dienstlichen Anweisungen, ohne eigene Denkweisen sowie Ideen zu entwickeln und einzubringen. Zudem weisen sie eine geringe Bindung zum Unternehmen auf. Die restlichen 15\% gaben an, keine emotionale Bindung zum Unternehmen zu besitzen und innerlich schon gekündigt zu haben. Die Folgen einer solchen innerlichen Kündigung, spiegeln auch die Fehlzeiten sowie die Fluktuation wieder, woraus wiederum volkswirtschaftlich relevante Kosten entstehen. Diese belaufen sich jährlich,

\footnotetext{
${ }^{1}$ www.zitate.de, Zitate Motivation, online: [URL] http://www.zitate.de/kategorie/Motivation, Stand 05.05.2015

${ }^{2}$ Vgl. Laufer, Hartmut: Praxis erfolgreicher Mitarbeitermotivation S. 56
} 
laut des Engagement Index Deutschland 2014, auf schätzungsweise 73-95 Milliarden Euro. $^{3}$

Dabei spielt die Mitarbeitermotivation eine immer wichtiger werdende Rolle in der heutigen Zeit. Die Angestellten eines Unternehmens sollen motiviert sein und von ihrem Vorgesetzten auch motiviert werden. Nur so kann sich eine Bindung zum Unternehmen aufbauen.

Dies setzt eine mitarbeiter- und bedürfnisorientiertere Führung der Vorgesetztenebene voraus, als sie noch vor einigen Jahrzehnten der Fall war. Wer davon ausgeht, dass Führungskräfte die Angestellten durch ihre Machtposition oder das Anordnen und Kontrollieren von Arbeitsaufgaben führen können, wird schnell bemerken, dass Mitarbeiterführung nie so schwierig und komplex war, wie heute. ${ }^{4}$

Die Motivation des Autors, sich mit dem Thema der Mitarbeitermotivation auseinander zu setzen, besteht in dem Interesse, herauszufinden, wie genau Führungspersonen ihre Angestellten motivieren können und welche Folgen mangelnde Motivation haben kann. Er sieht dies als Basis für seine später angestrebte Führungsposition an. Das Wissen über eine gute Mitarbeiterführung ist eine der wichtigsten Grundlagen einer jeden Führungsposition, denn die Motivation der Mitarbeiter steht und fällt mit dem Vorgesetzten. Ganz gleich ob es der Arbeitgeber bei Kleinunternehmen, oder die Führungskraft in Großunternehmen ist.

Im Folgenden soll anhand verschiedener wissenschaftlicher Theorien aufgezeigt werden, was die Angestellten eines Unternehmens wirklich benötigen, um ihrem Beruf glücklich nachgehen zu können.

\footnotetext{
${ }^{3}$ Vgl. Engagement Index Deutschland 2014, online: [URL] http://montua-partner.de/wpcontent/uploads/2015/04/Präsentation-zum-Engagement-Index-2014.pdf, Stand 05.05.2015

${ }^{4}$ Vgl. Laufer, Hartmut: Praxis erfolgreicher Mitarbeitermotivation S. 28
} 


\subsection{Grundlegende Fragestellung und Abgrenzung des Themas}

„Die Motivation ist das Zünglein an der Waage. Im Sport setzt man sie manchmal mit "geistiger Zähigkeit" gleich - wie könnte man sonst die zahlreichen Vorfälle in jeder

Saison erklären, bei denen eine Mannschaft eine andere verdientermaßen besiegt, doch ein paar Wochen später vom Verlierer niedergerungen wird? Fast immer schlägt die Motivation das große Talent. ${ }^{\text {w5 }}$ Norman R. Augustine

Dieses Zitat zeigt, wie wichtig Motivation ist - und das nicht nur im Sport. Die folgenden Kapitel werden sich dahingehend genauer mit dieser Thematik auseinandersetzen. Die Motivation, insbesondere die Mitarbeitermotivation, stellt ein sehr umfangreiches, aber dennoch interessantes und wichtiges Thema, sowohl jetzt als auch in Zukunft dar. Das veranschaulicht auch die Gallup Studie. Es bildet zudem die Grundlage einer jeden Führungsperson. Denn nur wer es schafft seine Mitarbeiter zu motivieren, wird zukünftig konkurrenzfähig bleiben und die Mitarbeiter an das Unternehmen binden können. Daher geht die Arbeit der Frage nach, wie Mitarbeiter durch den Arbeitgeber oder Vorgesetzten motiviert werden können. Ist es wirklich nur am Arbeitgeber, oder ist der unmittelbare Vorsetzte dafür verantwortlich, dass Mitarbeiter demotiviert sind? Müssen gegebenenfalls Grundstrukturen im Unternehmen verändert werden? Was ist überhaupt der Grund dafür, warum einige Mitarbeiter motiviert sind und andere wiederum nicht? Können Motivationsstrategien für alle Mitarbeiter gleich angewendet werden, oder verlangt dies die Betrachtung des Einzelnen? Antworten auf diese Fragen sollen sich im Rahmen der vorliegenden Arbeit ergeben und am Ende zusammenfassend beantwortet werden.

Um dies zu erforschen, jedoch gleichzeitig den Rahmen der Arbeit zu beachten, beschränkt sich der Autor auf die wichtigsten Inhalts- sowie Prozesstheorien der Vergangenheit. Die ausgewählten Theorien werden im Folgenden genauer vorgestellt und erläutert, um dem Leser einen Einblick in diese zu ermöglichen. Zudem werden anhand der Theorien die wichtigsten Erkenntnisse dieser für die Praxis herauskristallisiert und somit wichtige Anhaltspunkte für die Beantwortung der Fragestellung, sowie der aufgekommenen Fragen geliefert.

Des Weiteren wird aufgezeigt, was fehlende Mitarbeitermotivation für volkswirtschaftliche und gesundheitliche Folgen nach sich zieht und was die Ursache dafür ist, dass viele Mitarbeiter nicht richtig motiviert sind. Um dem Abhilfe zu schaffen, werden mittels

\footnotetext{
${ }^{5}$ www.zitate.de, Zitate Motivation, online: [URL] http://www.zitate.de/kategorie/Motivation?page=5, Stand 06.05 .2015
} 
der zuvor herauskristallisierten Erkenntnisse aus den Theorien, Anhaltspunkte geliefert, wie Mitarbeiter wirklich motiviert werden können und wie diese auch motiviert werden sollten.

Es wurde außerdem ein Fragebogen erstellt, der von den Mitarbeitern des Praktikumsbetriebes ausgefüllt wurde. Damit sollte herausgefunden werden, ob die zuvor beschriebenen Motivationstheorien mit der Praxis konform gehen und wie es mit der Motivation in dem Kleinunternehmen aussieht, um Handlungsempfehlungen ableiten zu können. Am Ende folgt ein Resümee der Bearbeitung und die Beantwortung, aller zu Beginn gestellten Fragen.

\subsection{Forschungsstand}

Die Thematik der Motivation ist ein umfassend erforschtes Gebiet, welches vor allem von amerikanischen Psychologen erforscht wurde. Diese lieferten die wichtigsten Erkenntnisse, welche bis heute noch großen Anklang finden. Die entstandenen Motivationstheorien lassen sich infolgedessen in fast jeder Personalführungs- oder Managementliteratur wiederfinden. Jeder der sich mit dieser Thematik beschäftigt, wird daher über Namen wie Maslow, Alderfer, Herzberg, McGregor, Vroom oder Locke und Latham stoßen, da diese die Motivationstheorien grundlegend geprägt haben. Daher gestaltete sich die Suche nach Literatur, welche diese Theorien beschreiben, als unkompliziert. Vielmehr war es eine Herausforderung zwischen der Vielzahl an Literatur diejenige zu finden, welche die Theorien am besten beschreiben konnte. Daher wurde hier auf mehrere Bücher zurückgegriffen, um einen umfänglichen Überblick der Motivationstheorien zu erhalten und um wichtige Erkenntnisse für die Praxis erlangen zu können.

In den sich daran anschließenden Kapiteln, wie Motivation von Mitarbeitern in der Praxis gelebt wird, welche Auswirkungen fehlende Motivation auf die Mitarbeiter und für die Unternehmen hat und wie Motivation erfolgen sollte, gestaltete sich die Suche nach geeigneter Literatur schon weitaus schwieriger. Über die Folgen von fehlender Motivation in der Praxis, ist keine Literatur vorhanden, welche das Thema ausführlich behandelt. Daher worden hier Statistiken wie z.B. die der Gallup Studie, der DAKGesundheitsreport oder der Gesundheitsreport der BARMER GEK herangezogen, um aus deren Auswertungen sowie der daraus resultierenden Erkenntnissen der Studien, Schlussfolgerungen zu ziehen. Literatur die beschreibt, was zumeist in Unternehmen gelebt wird, um Mitarbeiter zu motivieren und wie es anstelle dessen gemacht werden sollte, ist auch eher rar. Dennoch stieß der Autor auf ein Buch, dass für Jeden der sich ebenfalls für diese Thematik interessiert, sehr empfehlenswert ist. Aus diesem Grund, sollte sich jeder das Buch von Hartmut Laufer: „Praxis erfolgreicher Mitarbeitermotiva- 
tion" einmal zu Gemühte führen. Es beschreibt mit vielen Beispielen aus der Praxis, wie Unternehmen oder Führungskräfte Mitarbeiter zu motivieren versuchen und was falsch daran ist, oder was durch fehlende Kenntnis der Führungspersonen oder auch unbewussten Tätigkeiten dazu führen kann, dass Mitarbeiter demotiviert werden bzw. sind. Es wurde auch auf Expertenmeinungen aus Interviews von Dr. Reinhard K. Sprenger, einer der bekanntesten Führungsexperten Deutschlands und Prof. Dr. Gerald Hüther, ein Professor für Neurobiologie zurückgegriffen. Diese bekräftigen die Aussagen, welche auch Hartmut Laufer in seinem Buch erläutert und liefern zudem wichtige Erkenntnisse dafür, wie die Zukunft der Mitarbeitermotivation funktionieren soll und dass es für die Zukunft immer wichtiger wird, seine Angestellten für das was sie tun zu begeistern.

Prof. Gerald Hüther setzt sich auch für einen Kulturwandel der Unternehmen \& Organisationen, von der bisherigen Ressourcenausnutzungskultur, hin zu einer Potenzialentfaltungskultur dieser ein. Er zeigt anhand einiger Beispiele, welche Unternehmen schon einen messbaren Kulturwandel vollzogen haben, und welche auf dem Weg dahin sind. Dies soll für Andere ein Beispiel zur Umsetzung darstellen, denn daran lässt sich erneut erkennen, was wichtig ist, damit Mitarbeiter sich wohl fühlen und motiviert sind. ${ }^{6}$

\footnotetext{
${ }^{6}$ Vgl. Kulturwandel in Unternehmen \& Organisationen, online: [URL] http://www.kulturwandel.org/content/die-herausforderung/, Stand 08.05.2015
} 


\section{Theoretische Vorüberlegungen}

\subsection{Was ist Motivation?}

\subsubsection{Definition Motivation}

Wenn man sich mit der Thematik der Mitarbeitermotivation beschäftigt, stellt sich schnell heraus, dass es keine einheitliche Definition für das Wort Motivation gibt. Hartmut Laufer ist diesbezüglich folgender Auffassung: „Bei „Motivation“ handelt es sich um die Summe der Motive, die das Verhalten bzw. Denken oder Handeln einen Menschen aktuell bestimmen. [...]"

Laut Duden online, handelt es sich bei dem Begriff Motivation, um die "Gesamtheit der Beweggründe, Einflüsse, die eine Entscheidung, Handlung o. Ä. beeinflussen, zu einer Handlungsweise anregen. “8

Gemäß des Gabler Wirtschaftlexikons, ist Motivation, der „Zustand einer Person, der sie dazu veranlasst, eine bestimmte Handlungsalternative auszuwählen, um ein bestimmtes Ergebnis zu erreichen und der dafür sorgt, dass diese Person ihr Verhalten hinsichtlich Richtung und Intensität beibehält. [...] Der Begriff der Motivation wird oft auch im Sinn von Handlungsantrieben oder Bedürfnissen verwendet."

In der vorliegenden Arbeit wird Motivation als ein Zustand innerer Anregung zu einer bestimmten Handlung angesehen.

\subsubsection{Definition Demotivation}

Angelehnt an die Definition der Motivation, stellt Demotivation den Zustand innerer Abneigung gegenüber einer bestimmten Handlung dar.

\footnotetext{
${ }^{7}$ Vgl. Laufer, Hartmut: Praxis erfolgreicher Mitarbeitermotivation S. 34

${ }^{8}$ Duden online, Definition Motivation, online: [URL] http://www.duden.de/rechtschreibung/Motivation, Stand 08.05 .2015

${ }^{9}$ Gabler Wirtschaftslexikon, Definition Motivation, online: [URL]

http://wirtschaftslexikon.gabler.de/Definition/motivation.html, Stand 08.05.2015
} 


\subsubsection{Extrinsische Motivation}

Die Motivation lässt sich wissenschaftlich gesehen in zwei grundsätzliche Arten unterscheiden, die extrinsische und die intrinsische Motivation.

Wie der Titel schon andeutet, handelt es sich bei der extrinsischen Motivation um Einflüsse, die von außen auf die Motivation eines Menschen einwirken. Diese sind z.B. Geld, in Form von Gehaltserhöhungen oder Prämien, Beförderungen innerhalb eines Unternehmens, oder Statussymbole, wie ein schöner Dienstwagen. ${ }^{10}$ Die extrinsische Motivation beschreibt folglich die Verhaltensweisen einer Person, die in der Regel nicht spontan auftreten, sondern durch Aufforderungen und deren Erwartung auf Belohnung, oder Vermeidung von Konsequenzen zurückzuführen sind. Die Person wird nicht durch die Tätigkeit allein, sondern durch deren Begleitumstände motiviert. ${ }^{11}$

Die extrinsische Motivation kann infolgedessen als Zustand innerer Anregung ein bestimmtes Verhalten zu zeigen angesehen werden, mit der Erwartung, einer bestimmten daraus resultierenden Folge.

\subsubsection{Intrinsische Motivation}

Die intrinsische Motivation „bezieht sich auf einen Zustand, bei dem wegen eines inneren Anreizes, der in der Tätigkeit selbst liegt [...] gehandelt wird. Eine hohe intrinsische Motivation wird oft als Vorraussetzung für kreative Leistungen angesehen. [...] ${ }^{\text {“12 }}$

Im Gegensatz zur extrinsischen, beinhaltet die intrinsische Motivation, Neugier und Interesse an den unmittelbaren Gegebenheiten der Umwelt. Sie ist durch eine gewisse Ziellosigkeit gekennzeichnet, da kein Ergebnis erreicht werden soll, sondern die Ausführung selbst, gewissermaßen das Ziel darstellt. ${ }^{13}$

Es ist demnach ein Zustand innerer Anregung ein bestimmtes Verhalten zu zeigen, die aus einer zwanglosen, selbst erlebten Freude an einer Tätigkeit resultiert.

\footnotetext{
${ }^{10}$ Vgl. Göstl, Caterina: Mitarbeitermotivation S. 24

${ }^{11}$ Vgl. Motzkuhn, Mario: Wie geht Motivation wirklich? S. 6

${ }^{12}$ Gabler Wirtschaftslexikon, intrinsische Motivation, online: [URL] http://wirtschaftslexikon.gabler.de/Definition/intrinsische-motivation.html, Stand 08.05.2014

${ }^{13}$ Vgl. Motzkuhn, Mario: Wie geht Motivation wirklich? S. 6
} 


\subsection{Arten der Motivationstheorien}

\subsubsection{Inhaltstheorien}

Die Motivationstheorien werden in zwei Formen unterschieden. Zum einen in Inhaltstheorien und zum anderen in Prozesstheorien.

Die Inhaltstheorien gehen dabei der Frage nach, wodurch Motivation entsteht, was ein Mensch anstrebt und welche Bedürfnisse zu einem bestimmten Verhalten des Menschen führen. Zu den wichtigsten Inhaltstheorien gehören die Bedürfnistheorie nach Maslow, die ERG-Theorie nach Alderfer, die Zwei-Faktoren-Theorie nach Herzberg, sowie die X-Y-Theorie von McGregor. Diese Theorien werden nachfolgend erläutert, da sie wichtige Erkenntnisse für die Praxis liefern. ${ }^{14}$

\subsubsection{Prozesstheorien}

Die Prozesstheorien hingegen zeigen auf, wie Motivation entsteht und wie dieser Prozess abläuft. Die VIE-Theorie nach Vroom, sowie die Zielsetzungstheorie nach Locke und Latham, zählen zu den wichtigsten Prozesstheorien. Nachfolgend werden diese genauer dargestellt, da sie wie auch die Inhaltstheorien, wichtige Erkenntnisse für die Praxis liefern. ${ }^{15}$

\subsection{Motivationstheorien}

\subsubsection{Bedürfnistheorie nach Maslow}

Abraham $\mathrm{H}$. Maslow war ein amerikanischer Sozialpsychologe und zählt mit seiner Bedürfnistheorie, welche er im Jahr 1954 entwickelte, als Begründer und wichtigster Vertreter der Humanistischen Psychologie. ${ }^{16}$ Er war der Auffassung, dass Menschen nicht nur ihren materialistischen Trieben ausgeliefert seien, sondern dass diese aktiv an ihrer Existenz mitwirken und dabei stets nach höheren Idealen streben, um glücklich zu sein. Mit seiner vorausschauenden Erkenntnis entwickelte er eine bis heute oft zitierte und populäre Theorie. Diese hat sich über die Jahre hinweg als Klassiker be-

\footnotetext{
${ }^{14}$ Vgl. Stock-Homburg, Ruth: Personalmanagement S. 70

${ }^{15}$ Vgl. Stock-Homburg, Ruth: Personalmanagement S. 70

${ }^{16}$ Vgl. Laufer, Hartmut: Praxis erfolgreicher Mitarbeitermotivation S. 40
} 
währt und ist daher in fast jeder Managementliteratur wiederzufinden. ${ }^{17}$ Im Laufe der Jahre haben sich dementsprechend viele verschiedene Grafiken der Bedürfnishierarchie entwickelt. Trotz dass Maslows Thesen nicht empirisch belegt sind, finden sie angesichts inrer Anschaulichkeit und einfachen Logik, dennoch Anklang in der Unternehmenspraxis. ${ }^{18}$

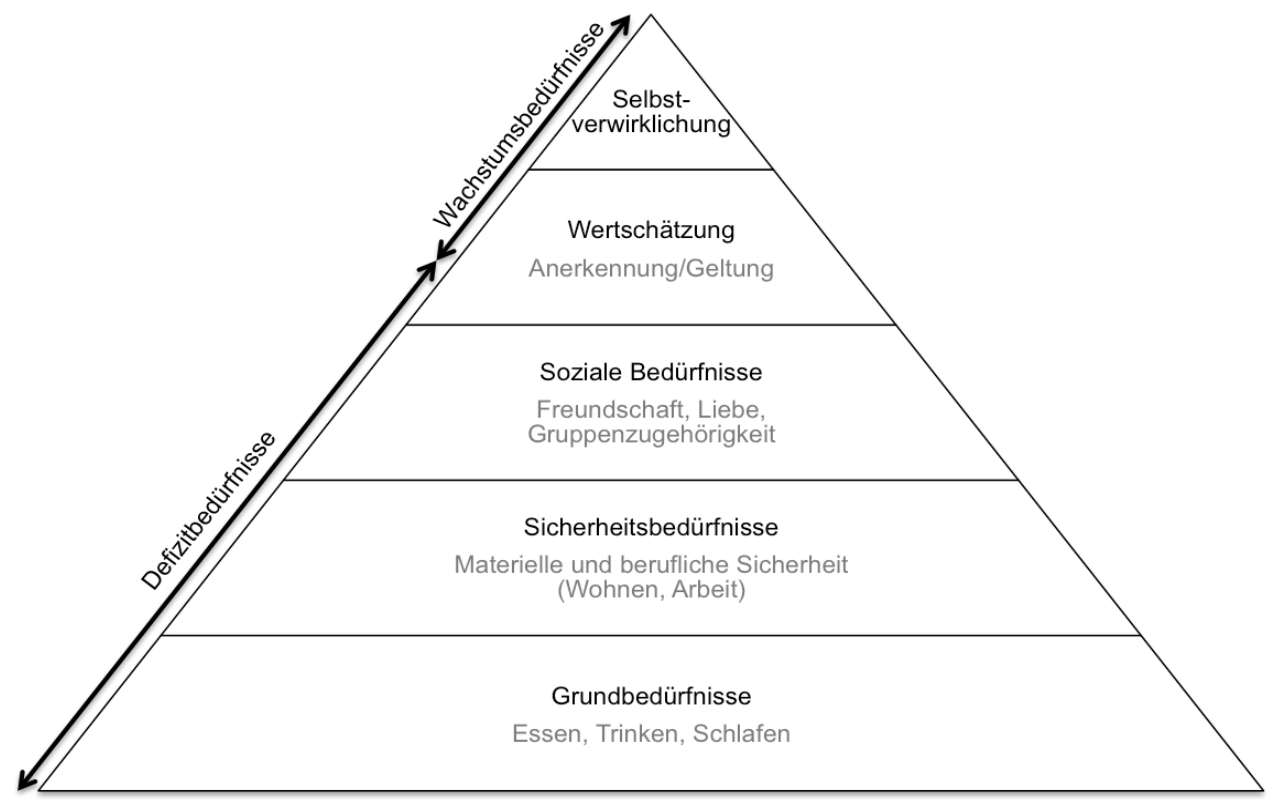

Abbildung 1: Maslowsche Bedürfnispyramide ${ }^{19}$

Die Abbildung veranschaulicht, dass Maslow die Bedürfnisse der Menschen in fünf Ebenen einteilt, welche hierarchisch nach ihrer Wichtigkeit angeordnet sind. Darunter zählen die Grundbedürfnisse, Sicherheitsbedürfnisse, sozialen Bedürfnisse sowie die Wertschätzung, als auch die Selbstverwirklichung.

Maslows Theorie zufolge geht ein Mensch schrittweise vor, um seine Bedürfnisse zu befriedigen. Dementsprechend werden als Erstes die Grundbedürfnisse eines Menschen, wie Speis und Trank befriedigt. Erst wenn diese erfüllt sind, strebt der Mensch das nächst höhere, das Sicherheitsbedürfnis an. Das bedeutet, dass stets das untere und damit bedeutendere Bedürfnis befriedigt werden muss, ehe man sich dem nächst höheren widmet. ${ }^{20}$ Die Theorie besagt jedoch nicht, dass die Befriedigung einer Bedürfnisebene für immer anhält. Sollte eine Person z.B. seine Wohnung oder Haus verlieren (Sicherheitsbedürfnis), steht für inn wieder an erster Stelle, sich um seine

\footnotetext{
${ }^{17}$ Vgl. Göstl, Caterina: Mitarbeitermotivation S. 35

${ }^{18} \mathrm{Vgl}$. Motzkuhn, Mario: Wie geht Motivation wirklich? S. 10

${ }^{19}$ Bildquelle: www.worklifecompetence.com/category/arbeitsorga/

${ }^{20}$ Vgl. Motzkuhn, Mario: Wie geht Mitarbeitermotivation wirklich? S. 9
} 
Grundbedürfnisse zu kümmern. Sobald diese sichergestellt sind, wird sich die Person erneut der nächst höheren Bedürfnisebene widmen, und nach einer neuen Wohnung suchen. ${ }^{21}$

Maslow unterteilt die fünf Bedürfnisebenen zudem in zwei Kategorien, die der Defizitund die der Wachstumsbedürfnisse. Sollten die Defizitbedürfnisse einer Person, zu denen die unteren vier Ebenen zählen, nicht befriedigt werden können, führt dies zur Unzufriedenheit und damit zu einem Mangelzustand an Motivation, sowie einer daraus folgenden psychischen Anspannung. Dies kann sogar zu einem krankheitsähnlichen Zustand führen. Die Wachstumsbedürfnisse, welche der Ebene der Selbstverwirklichung zugeteilt werden können, führen bei Befriedigung dazu, dass ein Mensch zufrieden und dadurch auch motiviert ist. ${ }^{22}$

Die Theorie lässt sich demzufolge auch auf die Arbeitswelt anwenden. Grundbedürfnisse sind in diesem Sinne nicht mehr Essen und Trinken, sondern das Gehalt oder die Arbeitsbedingungen einer Person. Sicherheit sieht der Angestellte z.B. in einem Kündigungsschutz, der Sozialversicherung, oder auch in der dauerhaften Gewährleistung seines Arbeitsplatzes. Soziale Bedürfnisse werden durch ein gutes Betriebsklima gefördert, im Sinne von Harmonie zwischen den Mitarbeitern. Die Wertschätzung erfährt ein Angestellter, durch die Anerkennung seiner Arbeit seitens des Vorgesetzten. Die höchste Bedürfnisebene der Selbstverwirklichung kann erreicht werden, indem ein Angestellter seine Arbeit nicht als Beruf sieht, sondern als seine Berufung.

Anhand der Bedürfnishierarchie Maslows, lassen sich folglich einige wichtige Erkenntnisse für die Motivation von Angestellten ableiten. Gesetz dem Fall, dass die Defizitbedürfnisse eines Angestellten nicht befriedigt werden, wie z.B. ein angemessenes Gehalt, die Sicherheit des Arbeitsplatzes oder auch Kollegen, mit denen man sich versteht, werden die Angestellten nicht motiviert sein und ihre Arbeit schlimmstenfalls nur auf das Nötigste beschränken. Dies kann jedoch auch geschehen, wenn ein Angestellter weiß, dass ein Kollege für die gleiche Tätigkeit ein höheres Gehalt erhält, oder das Gehalt in der Branche allgemein höher ist.

Aus diesem Grund müssen die Defizitbedürfnisse befriedigt sein, um zu erreichen, dass die Angestellten zumindest nicht demotiviert sind. Daher bedarf es nicht nur einer materiellen Vergütung oder sozialen Sicherheit, um einen Angestellten zu motivieren. Es sind auch immaterielle Anreize notwendig, wie die Wertschätzung der Arbeit, ein ehrliches Interesse an der Persönlichkeit, oder auch das Einräumen eines größeren

\footnotetext{
${ }^{21}$ Vgl. Laufer, Hartmut: Praxis erfolgreicher Mitarbeitermotivation S. 42

${ }^{22}$ Vgl. Motzkuhn, Mario: Wie geht Motivation wirklich? S. 9
} 
Handlungsspielraumes, sodass der Angestellte sein Bedürfnis nach Wertschätzung und Selbstverwirklichung befriedigen kann. ${ }^{23}$

\subsubsection{ERG-Theorie nach Alderfer}

Clayton P. Alderfer hatte die Absicht, die Bedürfnistheorie von Maslow empirisch zu belegen, gelang jedoch zu der Ansicht, dass eine eindeutige Abgrenzung der einzelnen Bedürfnisebenen nicht möglich ist. Daraufhin fasste er einzelne Bedürfnisse in bestimmte Gruppen zusammen und reduzierte die Ebenen dadurch von fünf auf drei. Er teilte sie in Existenzbedürfnisse (existence needs), Beziehungsbedürfnisse (relatedness needs) sowie Wachstumsbedürfnisse (growth needs) neu ein. Aus diesem Grund wird sie auch als ERG-Theorie bezeichnet und stellt eine Weiterentwicklung der von Maslow entwickelten Bedürfnistheorie dar.

Die Existenzbedürfnisse enthalten dabei grundlegende Bedürfnisse, welche sich nach Maslow in den zwei Ebenen der Grund- sowie Sicherheitsbedürfnisse wiederfinden. Die nach Maslow definierten Sozialen Bedürfnisse und die Wertschätzung, fasst Alderfer als Beziehungsbedürfnisse zusammen. Die Ebene der Selbstverwirklichung ist dem Bereich der Wachstumsbedürfnisse zuzuordnen. ${ }^{24}$

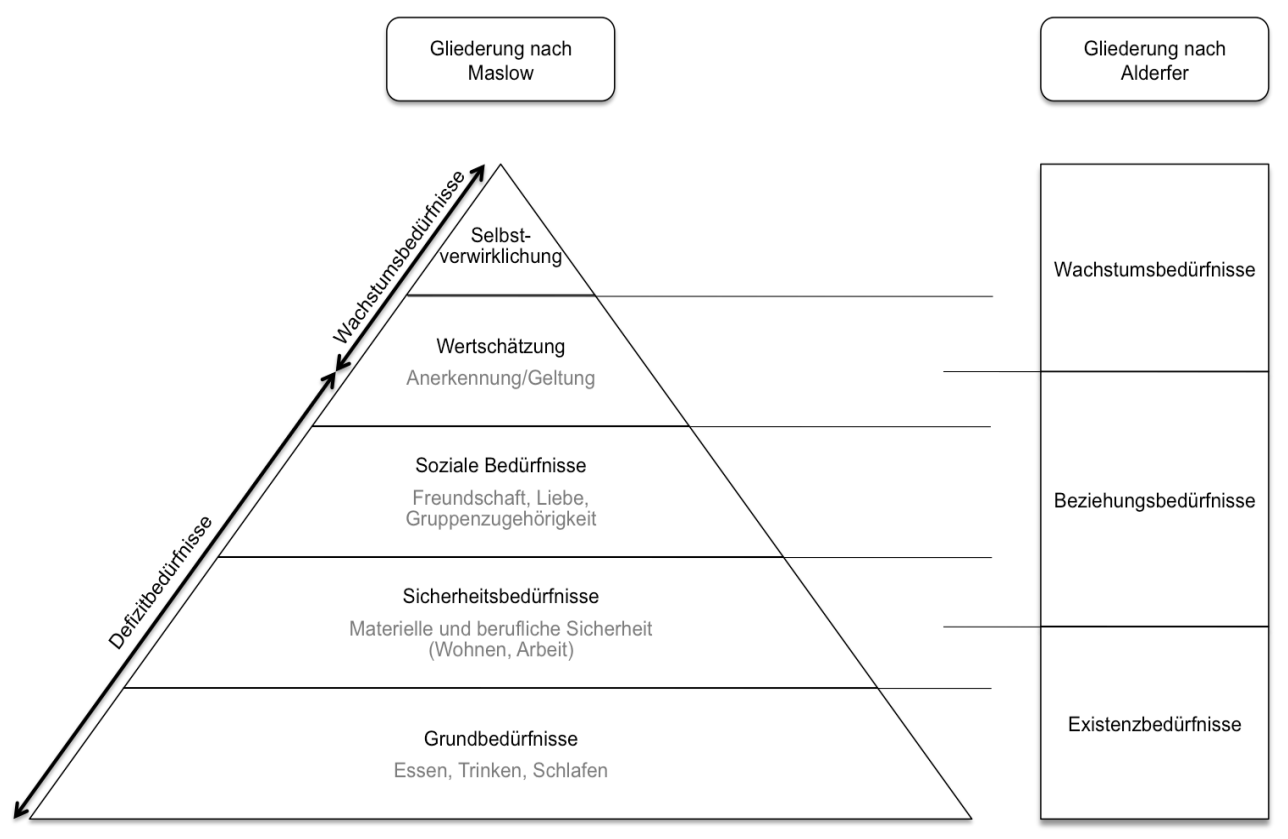

Abbildung 2: ERG-Theorie im Vergleich zur Bedürfnispyramide ${ }^{25}$

\footnotetext{
${ }^{23}$ Vgl. Göstl, Caterina: Mitarbeitermotivation S. 39

${ }^{24}$ Vgl. Stock-Homburg, Ruth: Personalmanagement S. 73

${ }^{25}$ Bildquelle: www.grundlagen-der-unternehmensfuehrung.de
} 
Den entscheidenden Unterschied bildet jedoch nicht die Neueinteilung der Bedürfnisse nach Alderfer, sondern seine Annahme. Diese besagt, dass nicht nur ein Bedürfnis, sondern mehrere Bedürfnisse zur selben Zeit angestrebt werden können. Sollte des Weiteren ein Bedürfnis einer Ebenen nicht befriedigt sein, führt auch dies nicht dazu, dass ein Mitarbeiter unmotiviert oder unzufrieden ist. Vielmehr kann es zu den vier folgenden Handlungsweisen führen. ${ }^{26}$

Nach der Frustrations-Hypothese verspürt ein Mensch Frustration, sobald er ein Bedürfnis nicht befriedigen kann. Demzufolge strebt er danach, dieses zufrieden zu stellen. Bei der Frustrations-Regressions-Hypothese, verstärkt sich das Verlangen ein niedrigeres Bedürfnis zu befriedigen, wenn ein bestimmtes Bedürfnis nicht befriedigt werden kann. Im Gegenzug dazu, führt dies bei der Frustrations-ProgressionsHypothese, zu einer Verstärkung des eigentlichen Bedürfnisses. Das Verlangen höhere Bedürfnisse zu befriedigen, kann dadurch bekräftigt werden. Wird ein höheres Bedürfnis durch die Befriedigung eines anderen aktiviert, so spricht man von der Befriedigungs-Progressions-Hypothese.

Für die Praxis lässt sich ableiten, dass ein jeder Mitarbeiter bezüglich der Befriedigung seiner Bedürfnisse als Individuum gesehen werden muss. Denn jeder reagiert anders auf die Befriedigung, oder auch mangelhafte Befriedigung seiner Bedürfnisse. Es ist Aufgabe der Führungsebene seinen Mitarbeitern Möglichkeiten und Chancen zu bieten, ihre jeweiligen Bedürfnisse zu erfüllen. ${ }^{27}$

\subsubsection{Zwei-Faktoren-Theorie nach Herzberg}

Neben der Bedürfnistheorie von Maslow zählt die Zwei-Faktoren-Theorie zu den bekanntesten Inhaltstheorien, welche im Jahr 1959 von Herzberg entwickelt wurde. Frederick I. Herzberg war ein amerikanischer Professor für Arbeitswissenschaften und fand durch umfangreiche Befragungen Beruftätiger heraus, dass die Arbeitszufriedenheit in zwei unterschiedliche Arten eingeteilt werden kann - die Hygienefaktoren und die Motivatoren. Aus diesem Grund, bezeichnete er seine Erkenntnis als Zwei-FaktorenTheorie. $^{28}$

Hygienefaktoren, auch Stabilisatoren genannt, führen bei einem Defizit zu einer Unzufriedenheit. Andererseits führen sie bei Vorhandensein nicht zur Zufriedenheit, sondern lediglich zu einem Zustand des nicht unzufrieden seins. Zu den Faktoren gehören im Wesentlichen Rahmenbedingungen der Arbeit, wie z.B. eine gerechte Bezahlung, die

\footnotetext{
${ }^{26}$ Vgl. Motzkuhn, Mario: Wie geht Motivation wirklich? S. 11

${ }^{27}$ Vgl. Stock-Homburg, Ruth: Personalmanagement S. 73

${ }^{28}$ Vgl. Laufer, Hartmut: Praxis erfolgreicher Mitarbeitermotivation S. 44
} 
Sicherheit des Arbeitsplatzes, angemessene Sozialleistungen, oder eine gute zwischenmenschliche Beziehung zu den Arbeitskollegen.

Im Gegensatz zu den Hygienefaktoren, kann durch das Vorhandensein von Motivatoren, ein Zustand der Zufriedenheit erreicht werden. Bleiben diese Faktoren aus, führt dies nicht zu einer Unzufriedenheit, sondern lediglich dazu, nicht zufrieden zu sein. Zu den Faktoren zählen unter anderem die Anerkennung durch Vorgesetzte, interessante Arbeitsinhalte, Aufstiegsmöglichkeiten oder selbstständiges Arbeiten. ${ }^{29}$

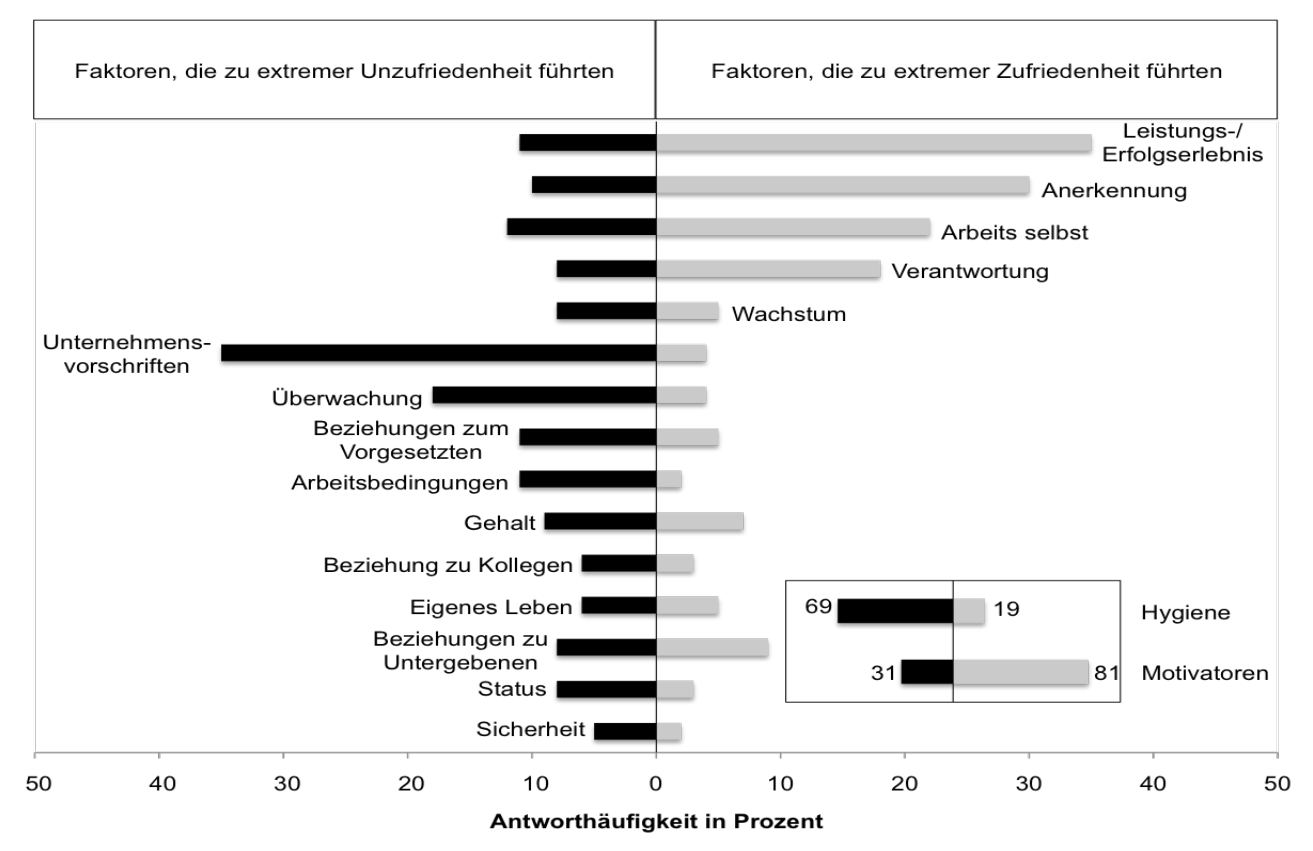

Abbildung 3: Hygienefaktoren und Motivatoren ${ }^{30}$

Anhand der Zwei-Faktoren-Theorie nach Herzberg, lässt sich für die Praxis die Schlussfolgerung ziehen, dass die Hygienefaktoren bedingungslos zu gewährleisten sind, sodass ein Aufkommen von Unzufriedenheit vermieden werden kann. Darüber hinaus sollte Wert auf den Ausbau der Motivatoren gelegt werden, da nur diese Motivationskräfte freisetzen und für eine echte Arbeitszufriedenheit sorgen können. ${ }^{31}$

Dennoch bleibt die Theorie nicht von Kritik verschont, da die Einteilung in Hygienefaktoren und Motivatoren zu schwammig sei. Demnach konnten Motivatoren häufiger mit Unzufriedenheit in Verbindung gebracht werden, als Hygienefaktoren. Des Weiteren

\footnotetext{
${ }^{29}$ Vgl. Motzkuhn, Mario: Wie geht Motivation wirklich? S. 12

${ }^{30}$ Bildquelle: www.grundlagen-der-unternehmensfuehrung.de

${ }^{31}$ Vgl. Göstl, Caterina: Mitarbeitermotivation S. 43
} 
waren Versuche, die Ergebnisse von Herzberg mit anderen Methoden zu wiederholen, größtenteils erfolglos. ${ }^{32}$

\subsubsection{X-Y-Theorie von McGregor}

Die von Douglas McGregor, einem amerikanischen Professor für Management entwickelte X-Y-Theorie, beschreibt zwei völlig verschiedene Menschenbilder der Arbeitswelt, welche er als Theorie $X$ und Theorie $Y$ bezeichnet. Diese stellte er in seinem, im Jahre 1960 erschienen Buch, "The Human Side of Enterprise“ vor. McGregor beabsichtigte damit, den Menschen nicht weiter nach Theorie X, sondern nach Theorie $Y$ zu betrachten und dementsprechend zu führen.

Die Theorie X stellt das negative Menschenbild dieser zwei Theorien dar. Der Mensch ist laut dieser von Geburt an faul, ohne jeglichen Ehrgeiz, oder dem Willen etwas zu leisten. Außerdem besitzt er keine Eigeninitiative oder Verantwortungsbewusstsein. Vielmehr verabscheut er jede Form von Arbeit. ${ }^{33}$ "Er muss gezwungen, kontrolliert und notfalls bestraft werden, um die für die Gesellschaft erforderlichen Arbeitsleistungen zu erbringen." ${ }^{34}$

Die Theorie $Y$ sieht den Menschen hingegen als das ganze Gegenteil. Sie beschreibt inn als einen arbeits- und leistungsbereiten, sowie verantwortungsbewussten und selbstständigen Menschen. Er ist intrinsisch motiviert und benötigt daher auch keine extrinsischen Einwirkungen, wenn er sich mit seiner Arbeit identifizieren kann und inm diese als sinnvoll erscheint. Bei eventuell auftretenden Problemen, versucht er diese daher auch eigenständig zu lösen.

Da die Unternehmen jedoch zu oft von dem falschen Menschenbild ausgehen, sprich jenes der Theorie $\mathrm{X}$, richten sie demzufolge ihre Motivationsstrategien falsch aus. Das kann folglich zu einer Demotivation der Angestellten führen. Auch Hartmut Laufer, welcher das MENSOR Institut für Managemententwicklung und systematische Organisationsberatung in Berlin leitet, schriebt in seinem Buch „Unter den heutigen Bedingungen kann man Mitarbeiter aber nur mit einer Grundeinstellung gemäß der Theorie $Y$ zu echtem, nachhaltigem Engagement und überdurchschnittlichen Leistungen führen. “35

\footnotetext{
${ }^{32}$ Vgl. arbeitzufriedenheit.net, Zwei-Faktoren-Theorie, online: [URL] http://arbeitszufriedenheit.net/herzbergs-zwei-faktoren-theorie/, Stand 13.05.2015

${ }^{33}$ Vgl. Göstl, Caterina: Mitarbeitermotivation S. 47

${ }^{34}$ Laufer Hartmut: Praxis erfolgreicher Mitarbeitermotivation S. 38

${ }^{35}$ Laufer, Hartmut: Praxis erfolgreicher Mitarbeitermotivation S. 39
} 
Auch die X-Y-Theorie bleibt nicht von Kritik verschont, da man nicht von einem rein nach Theorie $X$, oder rein nach Theorie $Y$ ausgerichteten Menschen ausgehen kann. Dessen war sich McGregor jedoch bewusst und arbeitete an einer Theorie Z, welche das Beste aus beiden Theorien vereinen sollte. McGregor schaffte es jedoch nicht seine Theorie fertig zu stellen, da er währenddessen verstarb. Es gelang inm jedoch erste Ansätze herausstellen, wobei laut Theorie Z, ein Gleichgewicht von extrinsischen und intrinsischen Anreizsystemen geschaffen werden, und den Angestellten Eigenverantwortung übertragen werden soll. Zusätzlich soll ihnen Anteilnahme an Entscheidungsprozessen gewährt werden. ${ }^{36}$

\subsubsection{Valenz-Instrumentalitäts-Erwartungstheorie nach Vroom}

Victor H. Vroom ist der Entwickler der VIE-Theorie, eine der am weitesten verbreiteten Prozesstheorien. ${ }^{37}$ Laut Vroom bedarf es drei Bedingungen, die erfüllt sein müssen, damit Motivation entstehen kann - Valenz, Instrumentalität sowie Erwartung. Diese verschafften seiner Theorie gleichzeitig ihren Namen. Die Valenz ist der individuelle Wert einer Handlung, welche positiv oder auch negativ sein kann. Die Erwartung, in der nachfolgenden Abbildung auch Anstrengungs-Leistungs-Erwartung genannt, beschreibt die subjektive Wahrscheinlichkeit, dass eine erhöhte Anstrengung auch zu der erhofften Leistung führt. Instrumentalität, ebenso auch Leistungs-Ergebnis-Erwartung, beschreibt die subjektive Wahrscheinlichkeit, dass die Leistung zu dem angestrebten Ergebnis führt. Nur wenn diese 3 Faktoren gegeben sind, kann laut Vroom eine Arbeitsmotivation entstehen. ${ }^{38}$

\footnotetext{
${ }^{36}$ Vgl. Göstl, Caterina: Mitarbeitermotivation S. $48 \mathrm{ff}$.

${ }^{37}$ Vgl. Stock-Homburg: Personalmanagement S. 80

${ }^{38}$ Vgl. Motzkuhn, Mario: Wie geht Motivation wirklich? S. 15
} 


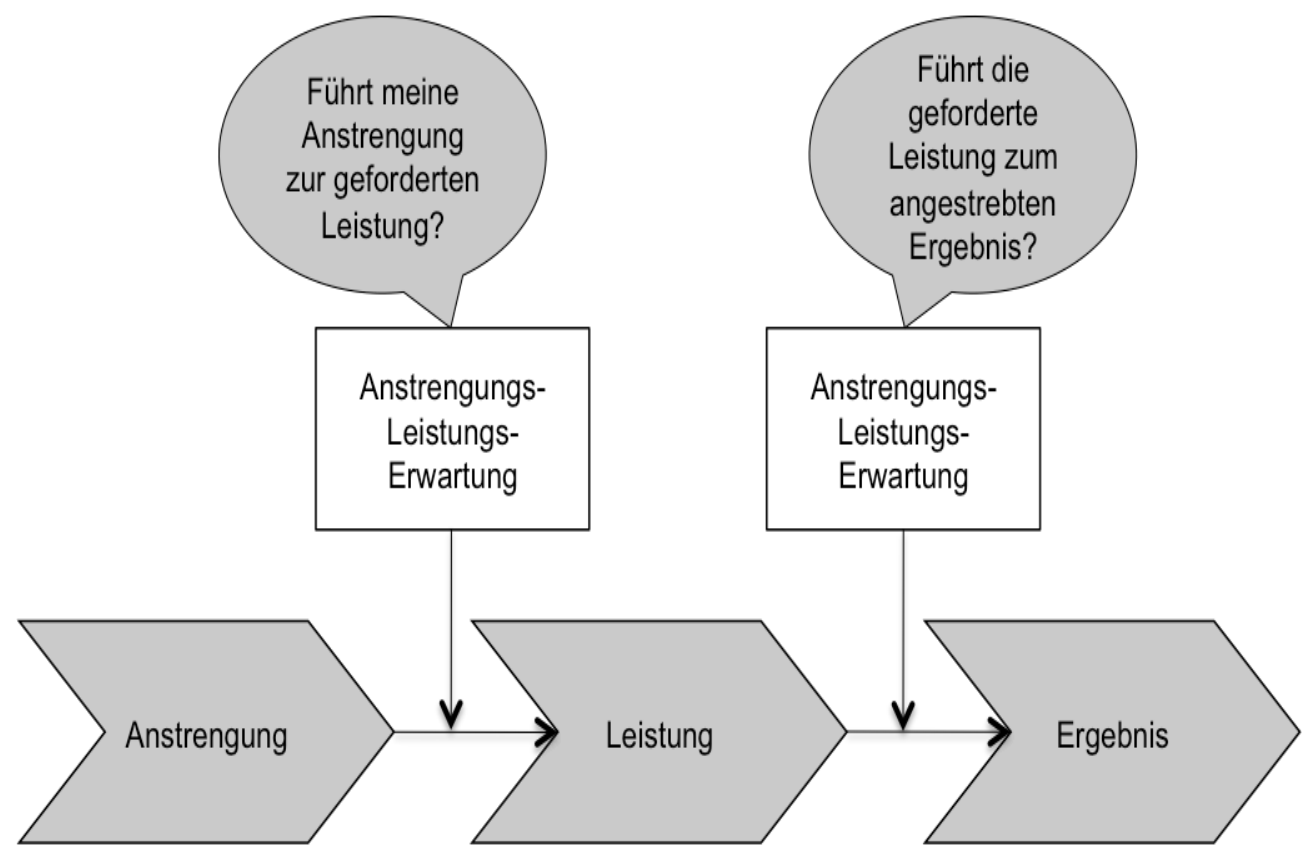

Abbildung 4: Konzept der VIE-Theorie nach Vroom $^{39}$

Das folgende Beispiel soll noch einmal der Veranschaulichung der Theorie dienen.

Ein Mitarbeiter hat sich ein Grundstück gekauft und baut nun darauf ein Haus. Um dies finanziell stemmen zu können, strebt er ein hohes Einkommen an (Ergebnis). Es ist inm sehr wichtig, dieses Ziel zu erreichen (hohe Valenz). Er weiß, dass er das angestrebte Einkommen, nur durch eine Beförderung erlangen kann (Leistung). Die Instrumentalität oder auch Leistungs-Ergebnis-Erwartung ist gegeben, da der Mitarbeiter weiß, dass eine Beförderung zu mehr Einkommen führt. Um befördert zu werden, wird der Mitarbeiter seine Leistungsergebnisse verbessern und auch länger arbeiten (Anstrengung). Die Erwartung oder auch Anstrengungs-Leistungs-Erwartung ist gegeben, da ihm bewusst ist, dass eine bessere und auch längere Arbeit bzw. Arbeitszeit zu einer Beförderungen führen kann.

Die Valenz einer Person kann sich im Laufe der Zeit, aufgrund von Lebensumständen (Familie) oder Karrierephasen ändern. Ist der Mitarbeiter, wie in dem soeben beschriebenen Beispiel, nach einer gewissen Zeit fertig mit dem Hausbau und hat irgendwann Kinder, stellt eine Beförderung für inn keinen Anreiz mehr da, da er dann zwar über ein höheres Einkommen verfügt, aber durch die Beförderung, aufgrund von längeren Arbeitszeiten, weniger Zeit für seine Familie aufbringen kann.

Die VIE-Theorie wird auch kritisch betrachtet, da Personen kein maximal optimiertes, sondern persönlich zufriedenstellendes Ergebnis anstreben und nicht ausschließlich

\footnotetext{
${ }^{39}$ Bildquelle: www.grundlagen-der-unternehmensfuehrung.de
} 
rational handeln, wovon jedoch Vroom ausging. Dennoch lässt sich für die Praxis die Schlussfolgerung ziehen, dass es wichtig ist, die Bedürfnisse der Mitarbeiter zu identifizieren und innen dahingehen Anreize zu schaffen, um diese Bedürfnisse befriedigen zu können. Nur so kann es gelingen Mitarbeiter zu motivieren. ${ }^{40}$

\subsubsection{Zielsetzungstheorie nach Locke und Latham}

Die Zielsetzungstheorie von Edwin Locke und Gary Latham setzt sich, wie es der Name bereits verlauten lässt, mit dem Prozess der Zielsetzung auseinander. Dabei kamen die beiden Amerikaner zu der Schlussfolgerung, dass ein präzise definiertes Ziel, mit einem herausfordernden Schwierigkeitsgrad, zu einer besseren Leistung und damit verbunden Motivation führt, als eine zu vage Zielformulierung in Verbindung mit einem, die Person unterfordernden Schwierigkeitsgrad. ${ }^{41}$

Zusammenhang zwischen Zielschwierigkeit und Leistung (Locke \& Latham, 1984)

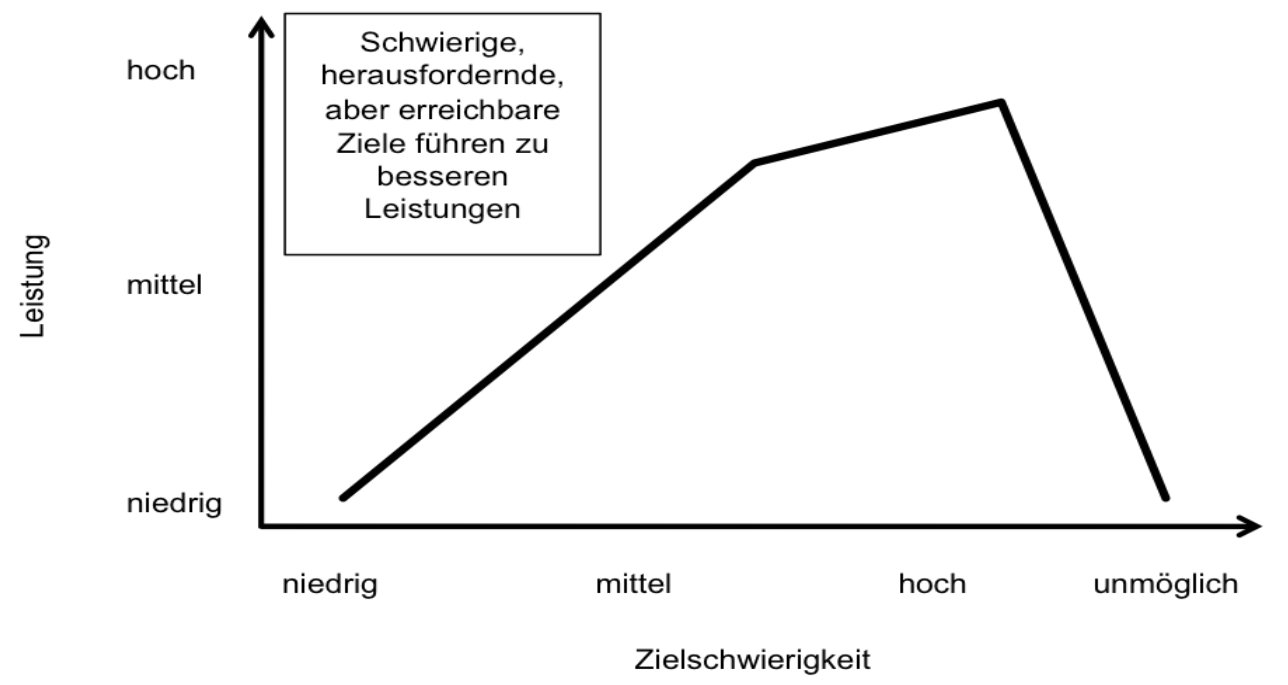

Abbildung 5: Zielsetzungstheorie nach Locke und Latham ${ }^{42}$

Wie stark die Motivation ausgeprägt ist, hängt von 3 Faktoren ab. Dem Schwierigkeitsgrad eines Ziels, einer exakten Zielvorgabe sowie der Akzeptanz und der Identifikation mit dem Ziel.

\footnotetext{
${ }^{40}$ Vgl. Stock-Homburg: Personalmanagement S. $81 \mathrm{ff}$.

${ }^{41} \mathrm{Vgl}$. Deutsche Akademie für Management, Zielsetzungstheorie, online: [URL] https://www.akademiemanagement.de/fernstudium-management/glossar-managementtheorien/zielsetzungstheorie, Stand 15.05.2014

42 Bildquelle: www.cobocards.com
} 
Um diese Faktoren auch wirkungsvoll umzusetzen zu können, bedarf es einiger Beachtung. Wirkt ein Ziel für die Person z.B. als ungreifbar, führt es dazu, dass die Leistung, und demzufolge auch die Motivation nachlässt. Infolgedessen fällt es der Person auch schwer, sich mit dem Ziel zu identifizieren und dieses zu akzeptieren, da die Erreichung als unrealistisch angesehen wird. Um ein Ziel dann noch definiert vorzugeben, empfiehlt es sich, sich an der SMART-Regel von Peter Ferdinand Drucker zu orientieren. Demnach müssen Ziele spezifisch, messbar, attraktiv, realistisch sowie terminiert sein. Werden diese soeben geschilderten Sachenverhalte beachtet, wirkt sich dies positiv auf die Motivation aus.

Für die Praxis ergibt sich anhand der Zieltheorie die wichtige Erkenntnis, dass ein zu erreichendes Ziel präzise formuliert, und im besten Fall mit der betroffenen Person gemeinsam gesetzt werden sollte. Dies sollte erfolgen, damit eine Identifikation sowie Akzeptanz mit und zu dem Ziel hergestellt wird und es zudem als realistisch angesehen wird. Dadurch soll die größtmögliche Motivation zur Zielerreichung geschaffen werden. Es empfiehlt sich, ein regelmäßiges Feedback zu geben, da dies auch leistungssteigernd wirkt und somit noch eventuelle Anpassungen vorgenommen werden können, um das Ziel bestmöglich zu erreichen. ${ }^{43}$

\subsection{Zusammenfassung}

\subsubsection{Gemeinsamkeiten der Theorien}

Die aufgeführten Theorien zählen zu den bedeutendsten Inhalts- sowie Prozesstheorien, weswegen sie vom Autor ausgewählt wurden. Nach genauerer Betrachtung dieser lässt sich erkennen, dass diese sich in ihren Grundannahmen und Erkenntnissen nicht widersprechen. Vielmehr ergänzen sie einander, und das trotz der Tatsache, dass jede dieser Theorien die Entstehung der Motivation aus einem anderen Blickwinkel betrachtet. Dennoch werden die Theorien auch kritisch gesehen, da diese keine genauen Angaben liefern können, wie Motivation entsteht, da der Mensch aufgrund seiner komplexen, individuellen Persönlichkeitsstruktur ein untypisches Motivationsverhalten aufweist. Zudem werden in den Theorien Einflüsse, wie beispielsweise aus Religionen, die das Verhalten von Menschen beeinflussen können, ebenso nicht berücksichtigt.

Trotz alledem zeigen die Erkenntnisse der Theorien, dass Motivation nur individuell erfolgen kann und es keinen perfekten, sowie einheitlichen Weg gibt, Mitarbeiter zu motivieren. Die Theorien zeigen auch, dass Geld einen bestimmten Wert hat und es

\footnotetext{
${ }^{43}$ Vgl. Motzkuhn, Mario: Wie geht Motivation wirklich? S. $17 \mathrm{ff}$.
} 
wichtig ist. Jedoch kann durch dieses allein, keine dauerhafte Motivation erfolgen. Vielmehr sind es Faktoren wie Wertschätzung und Selbstverwirklichung, die ein Mitarbeiter benötigt. Für die Motivation eines Mitarbeiters ist demnach eine individuelle Betrachtung diesen und dessen Bedürfnissen von Nöten, um die richtigen Ansätze herauszufinden zu können. 


\section{$3 \quad$ Motivation in der wirtschaftlichen Praxis}

\subsection{Was wird in der Praxis gelebt?}

Wie die Zahlen des Engagement Index 2014 zeigen, sind die meisten Angestellten nicht motiviert und besitzen auch keinen Anreiz, eigene Ideen in das Unternehmen einzubringen und verrichten infolgedessen lediglich ihren "Dienst nach Vorschrift“. ${ }^{44}$ Doch wie wird Motivation von Mitarbeiter wirklich in der Praxis umgesetzt und was ist der Grund dafür, dass nur wenige Mitarbeiter wirklich motiviert sind ihre Arbeit zu verrichten? Gerade da die Thematik der Motivation sehr komplex ist, gibt es sehr viele Dinge, die man falsch macht, wenn auch zumeist unbewusst. Dem muss jedoch Abhilfe geschafft werden.

Ein Hauptgrund für demotivierte Mitarbeiter, oder das Fehlen von motivierten Mitarbeitern, ist in den meisten Fällen der unmittelbare Vorgesetzte dieser. Oft sind Führungskräfte für die fehlende Motivation der Mitarbeiter verantwortlich, da sie vielmals nicht über die nötigen Kenntnisse verfügen, ihre Mitarbeiter zu motivieren oder ihnen die dafür benötigten Freiräume, nicht gewährt werden. Die Mitarbeiter heutzutage zu motivieren, ist aufgrund des Wertewandels der letzten Jahrzehnte weitaus schwieriger, als es noch vor dieser Zeit der Fall war. ${ }^{45}$ Führungskräfte beschränken sich zu oft darauf, strikte Arbeitsaufgaben anzuordnen und diese genau zu kontrollieren, oder aufgrund ihrer Machtposition die Mitarbeiter durch Angst zu führen. Angst steigert aber weder die Leistungs- noch die Veränderungsbereitschaft der Mitarbeiter und wenn doch, bringt das lediglich kurzfristige Erfolge. ${ }^{46}$ Selbst wenn Führungskräfte versuchen, an das Pflichtbewusstsein ihrer Mitarbeiter zu appellieren, werden sie zumeist nur belächelt und bekommen schnell mit, dass dies in der heutigen Zeit keine große Wirkung erzielt.

Häufig sind auch die Unternehmen schuld, die es den Führungskräften unnötig schwer machen, ihre Mitarbeiter zu motivieren. Um Personalkosten zu senken, wurden in vielen Unternehmen teilweise ganze Führungsebenen gestrichen. Die Konsequenz daraus ist, dass viele Führungspersonen nun viel mehr untergeordnete Mitarbeiter haben, um welche sie sich kümmern müssen. Doch in Zeiten in denen hohe Leistungsanforde-

\footnotetext{
${ }^{44}$ Vgl. Engagement Index Deutschland 2014, online: [URL] http://montua-partner.de/wpcontent/uploads/2015/04/Präsentation-zum-Engagement-Index-2014.pdf, Stand 05.05.2015

${ }^{45}$ Vgl. Laufer, Hartmut: Praxis erfolgreicher Mitarbeitermotivation S. 28

${ }^{46}$ Vgl. Hüther, Gerald: Lufthansa Exclusive Interview, online: [URL] http://www.geraldhuether.de/populaer/veroeffentlichungen-von-gerald-huether/zeitschriften/lufthansa-exclusive/index.php, Stand 20.05.2015
} 
rungen und Terminzwänge herrschen, werden die Gelegenheiten zu persönlichen Gesprächen oder gemeinschaftlichen Aktivitäten immer mehr reduziert und es bleibt den meisten Führungspersonen kaum Zeit, sich einzelnen Mitarbeitern intensiver zu widmen. Sie sind daher gezwungen, sich auf das anweisen und kontrollieren ihrer Mitarbeiter zu beschränken. ${ }^{47}$ Dieser Meinung ist auch Reinhard K. Sprenger, einer der bekanntesten Führungsexperten Deutschlands. In einem Interview äußert er sich wie folgt: „Zwar wird immer die Bedeutung der Führungsarbeit betont, den Führungskräften aber kaum Zeit zugestanden, sich ernsthaft mit ihren Mitarbeitern zu beschäftigen. [...] Sie kämpfen um wichtige Kunden, arbeiten an Projekten, erstellen Präsentationen und versuchen, die Flut der E-Mails zu bewältigen. Dieser Spagat zwischen Teamführung und Sachkompetenz ist nicht schmerzfrei zu leisten." "48 Auch die Mitarbeiter, von denen ein Kreatives handeln gefordert wird, haben es durch Stressfaktoren wie z.B. Zeitmangel, aufgrund von Terminen, Überforderungen und damit einhergehenden Versagensängsten, sowie durch Kritik oder Vorwürfe schwer. Ihr Denken wird dadurch behindert, oder sogar Denkblockaden verursacht.

So fehlt es den Unternehmen oft auch an einer Führungsphilosophie. Diese ist jedoch notwendig, damit das Unternehmen als harmonisches Ganzes funktionieren kann. Zudem übt sie einen Einfluss darauf aus, wie die Menschen miteinander umgehen. Auch das Vertrauen der Mitarbeiter zu ihren Firmen und die Identifikation mit diesen, geht durch Fusionen von Unternehmen und den damit verbunden Umorganisierungen oder auch Stellenabbau, bzw. durch Insolvenzen verloren. Früher waren die Menschen meist ein ganzes Berufleben lang in einem Unternehmen, doch heute ist dem längst nicht mehr so. Viele Unternehmen sind immer mehr mit sich selbst und den Gewinnen, als mit ihren Kunden beschäftigt, und die Mitarbeiter können nicht mehr stolz sein, Teil der Firma zu sein. Dies hat negative Konsequenzen für die Mitarbeitermotivation. ${ }^{49}$ Gerald Hüther, ein Hirnforscher und Autor vieler wissenschaftlicher Bücher, sagte in einem Interview: „Welcher Mitarbeiter wird sich dafür begeistern, den Shareholdern ihre Aktiengewinne zu vergrößern? Der freut sich, wenn er für eine Firma arbeitet, die in der Öffentlichkeit und bei den Kunden einen guten Ruf hat. Geht es nur noch ums Geld, hat der Einzelne das Gefühl, auf mich kommt es nicht mehr an. Er sieht keinen

\footnotetext{
${ }^{47}$ Vgl. Laufer, Hartmut: Praxis erfolgreicher Mitarbeitermotivation S. $16 \mathrm{ff}$.

${ }^{48}$ Sprenger, Reinhardt K.: Interview Reinhardt K. Sprenger, online: [URL] http://www.karriere.de/unternehmen/ein-unternehmen-das-eine-suchanzeige-aufgeben-muss-hat-schonverloren-166108/2/, Stand 20.05.2015

${ }^{49}$ Vgl. Laufer, Hartmut: Praxis erfolgreicher Mitarbeitermotivation S. 24 ff.
} 
Sinn mehr, fühlt sich verwaltet. Es folgt ein Leistungsabfall und die innere Kündigung." 50

\subsection{Folgen fehlender Motivation der Mitarbeiter}

Es lässt sich feststellen, dass zu viele Sachen in Unternehmen, bezüglich der Mitarbeitermotivation, nicht optimal laufen. Die Folgen, die sich aufgrund von falschen Strategien, fehlender Kompetenz der Vorgesetzten, des Führungspersonals, oder wegen ihrer fehlenden Freiräume ergeben, sind weitaus gravierender als man es im ersten Moment annehmen mag. Laut des Engagement Index von Gallup, entstanden im Jahr 2014 allein in Deutschland, geschätzte volkswirtschaftliche Kosten in Höhe von 73-95 Milliarden Euro, aufgrund von innerlichen Kündigungen. Diese finanziellen Folgen resultieren aus Fehlzeit, Fluktuation, einer geminderten Leistungsbereitschaft und damit einhergehenden Qualitätsverlusten, sowie einem abnehmenden Innovationspotenzial, was auf mangelnde Motivation zurückzuführen ist.

Dadurch, dass sich Mitarbeiter nicht mit dem Unternehmen verbunden fühlen, weisen sie höhere Fehlzeiten z.B. aufgrund von Unwohlseins oder Krankheit auf, als Mitarbeiter, die eine starke Bindung zu ihrem Unternehmen besitzen. Die Mitarbeiter sind demzufolge auch eher bereit, die Firma zu verlassen und sich eine Andere zu suchen. Außerdem würden sie das Unternehmen nicht weiterempfehlen, was es dem Unternehmen erschwert, neue Mitarbeiter zu finden. Allein bei Krankschreibungen entstehen den Unternehmen Kosten, durch Lohn- und Gehaltsfortzahlungen, die unbesetzten Arbeitsplätze, oder durch Personalreserven (Springer), die ein Unternehmen in solchen Fällen einsetzt. ${ }^{51}$

Die Auswirkungen betreffen aber nicht nur Unternehmen, sondern auch die Menschen selbst. Das zeigt auch der jährlich veröffentlichte DAK-Gesundheitsreport. Während im Jahr 2009 noch Erkrankungen des Muskel-Skelett-Systems mit 21\% des Krankenbestandes, dicht gefolgt von Erkrankungen des Atmungssystems (19\%) und Verletzungen $(13,6 \%)$ an der Spitze standen, und gerade einmal 10,8\% des Krankenbestandes auf psychische Erkrankungen zurückgeführt werden konnte, sieht es heute ganz anders aus. Die Zahl der psychisch Erkrankten nahm über die Jahre immer mehr zu. So waren psychische Erkrankungen bereits 2012 erstmalig an zweiter Stelle mit 15\%. Im Jahr 2014 sogar schon mit 16,6\%. Dies zeigt, dass die Ausfälle, aufgrund von psychi-

\footnotetext{
${ }^{50}$ Hüther, Gerald: Lufthansa Exclusive Interview, online: [URL] http://www.geraldhuether.de/populaer/veroeffentlichungen-von-gerald-huether/zeitschriften/lufthansa-exclusive/index.php, Stand 20.05.2015

${ }^{51}$ Vgl. Engagement Index Deutschland 2014, online: [URL] http://montua-partner.de/wpcontent/uploads/2015/04/Präsentation-zum-Engagement-Index-2014.pdf, Stand 05.05.2015
} 
schen Erkrankungen, immer mehr zunehmen. Das belegen auch andere Krankenkassen, wie beispielsweise die BARMER GEK - die größte gesetzliche Krankenkasse Deutschlands. ${ }^{52}$

Diese Erkrankungen fallen teilweise auch auf die Führungspersonen bzw. die Unternehmen zurück. Damit die Arbeitssituation eines Mitarbeiters als gesundheitsgerecht und auch gesundheitsförderlich angesehen werden kann, werden Dinge wie eine gute Arbeitsplatzausstattung vorausgesetzt, um kurz- und langfristige Schädigungen zu verhindern. Außerdem sind interessante Arbeitsaufgaben sowie Handlungsspielräume notwendig und es müssen persönliche, als auch berufliche Entwicklungsperspektiven gegeben sein. Eine Einbeziehung in Entscheidungsprozesse, sowie die Annerkennung und Unterstützung durch die Vorgesetzten, sind dafür ebenfalls von Nöten. ${ }^{53}$

Dabei spielt auch die Führungsebene eine Rolle. Denn die Stimmung bzw. Laune der Führungsperson, ist auch für die Mitarbeiter von Bedeutung. Ist der Vorgesetzte ständig mies gelaunt, wird sich das auch auf die Mitarbeiter übertragen. Ist die Führungsperson beispielsweise ein Choleriker, kann das beim Angestellten gegebenenfalls auch Muskelanspannungen, Schwitzen, Herzklopfen oder einen erhöhten Blutdruck auslösen, was auf Dauer krank macht. Die Angst wiederum könnte dazu führen, dass die Leistungsfähigkeit blockiert wird und sich das Wohlbefinden sich stark verschlechtert.

Stattdessen können Gestaltungsspielräume und die Weiterentwicklung der Mitarbeiter gefördert werden, was auch das Betriebsklima erheblich prägen kann. Ein gutes Verhältnis zum Vorgesetzten ist nicht nur für die Motivation, sondern auch für ein gesundes arbeiten wichtig. ${ }^{54}$

\subsection{Wie können Mitarbeiter motiviert werden?}

Wichtige Anhaltspunkte hierfür liefern die zuvor vorgestellten Motivationstheorien. Anhand dieser lässt sich erkennen, was Mitarbeiter wirklich benötigen, um motiviert zu sein. Natürlich lassen sich die nachfolgenden „Beschreibungen“ auf unterschiedliche Branchen anwenden, doch im Nachfolgenden sollen dem Leser die allgemeinen Erkenntnisse dargestellt werden, um ein Grundverständnis zu entwickeln, wie Mitarbeiter motiviert werden können.

\footnotetext{
${ }^{52}$ Vgl. DAK-Gesundheitsreport 2015, online: [URL] http://www.dak.de/dak/download/Vollstaendiger_bundesweiter_Gesundheitsreport_2015-1585948.pdf, Stand 22.05.2015

${ }^{53}$ Vgl. Hurrelmann, Klaus; Klotz, Theodor; Haisch, Jochen: Lehrbuch Prävention und Gesundheitsförderung S. 333

${ }^{54}$ Vgl. Katrin Matyssek, Anne: Führung und Gesundheit S. $31 \mathrm{ff}$.
} 
Grundsätzlich sollte man nicht immer sofort davon ausgehen, dass Mitarbeiter nicht motiviert sind, wenn sie eine Aufgabe nicht zur vollster Zufriedenheit erledigen. Denn dies könnte auch daran liegen, dass ein Mitarbeiter motiviert ist, aber z.B. nicht über die zur Bewältigung der Aufgabe notwendigen Kenntnisse oder Fertigkeiten verfügt, um diese besser bewältigen zu können. Wird der Mitarbeiter infolgedessen dann auch noch bestraft, obwohl er seiner Aufgabe motiviert nachgegangen ist und diese lediglich aufgrund fehlender Kenntnisse oder Fertigkeiten nicht zufriedenstellend war, hat dies einen direkten Einfluss auf die Motivation des Mitarbeiters. Diese wird dann infolge der Bestrafung abnehmen, da selbst der motivierteste Mitarbeiter bei fehlenden Fähigkeiten, die er zu Bewältigung der Aufgabe benötigt, keine Höchstleistungen erbringen kann. Um dem entgegenzuwirken ist es wichtig, wie es auch die Erkenntnisse der Zielsetzungstheorie von Locke und Latham bestätigen, die Ziele mit dem Mitarbeiter zusammen festzulegen. Das heißt nicht, dass die festgelegten Ziele einfach sein sollen. Sie sollten den Mitarbeiter schon fordern, aber es lässt sich bei einer gemeinsamen Absprache erkennen, ob er dieses als machbar empfindet, oder von vornherein schon auf ihm fehlende Fähigkeiten hinweist.

Sollte man jedoch wissen, dass ein Mitarbeiter demotiviert ist, da man z.B. fehlende Kompetenzen ausschließen kann, müssen Motivationsanreize geschaffen werden. Dabei ist es wichtig, dass diese individuell auf den Mitarbeiter abgestimmt sind und somit auch auf ein unbefriedigtes Bedürfnis des Mitarbeiters stoßen, welches er dann befriedigen kann. Dies zeigte auch die Erkenntnis der Valenz-InstrumentalitätsErwartungstheorie nach Vroom. Es ist wichtig, dass der Mitarbeiter, von der inm gebotenen Maßnahme auch einen Nutzen hat. So kann sich ein Mitarbeiter z.B. nicht erfreuen, wenn ihm ein vorgezogener Feierabend als Belohnung angeboten wird, er damit aber nichts anfangen kann, da er auf seinen Kollegen warten muss. Dies gilt auch für Arbeitsaufgaben. Der Mitarbeiter muss die Chance erkennen, dass er mit der inm übertragenen Arbeit, ein persönliches Bedürfnis befriedigen kann. Man kann inm beispielsweise eine schwierige Aufgabe geben, dessen Resultat bei Bewältigung derer, eine höhere Anerkennung sein könnte.

Die aktuellen Bedürfnisse der Mitarbeiter zu kennen ist natürlich nicht selbstverständlich, da es oft nicht einfach ist, diese herauszufinden bzw. zu erkennen. Hierfür ist es wichtig, dass die Führungspersonen auch ein ehrliches Interesse zeigen und die Zeit aufbringen, sich mit den einzelnen Mitarbeitern intensiver auseinanderzusetzen. Merkt der Mitarbeiter, dass die Führungsperson wirklich Interesse an ihm zeigt, wirkt dies auch motivierend. Er bemerkt dadurch, dass es der Führungsperson auch um die Belange der Mitarbeiter geht, und nicht nur um Umsatzzahlen. Ist das aufgrund der zeitli- 
chen Umstände nicht möglich, wäre auch die direkte Frage, was der Mitarbeiter sich z.B. für eine geleistete Mehrarbeit wünschen würde, eine Möglichkeit. ${ }^{55}$

Was besonders für Klein- und Mittelsständische Unternehmen interessant sein sollte, ist das Thema Gehalt. Um es gleich vorwegzunehmen, eine dauerhafte Motivation, kann durch ein hohes Gehalt allein nicht gewährleistet werden. Vielmehr sind es, wie es sich anhand der Bedürfnistheorie nach Maslow oder der Zwei-Faktoren-Theorie von Herzberg schon entnehmen lässt, immaterielle Anreize, die eine dauerhafte Motivation schaffen und für eine echte Arbeitszufriedenhei, sowie ein daraus resultierendes langfristiges Mitarbeiterengagement sorgen. Immaterielle Anreize stellen z.B. interessante und mit Erfolgserlebnissen ausgestattete Arbeitsinhalte, ein selbstständiges und kreatives Handeln, aber auch Annerkennung, Wertschätzung und ein ehrliches Interesse seitens der Führungsperson dar. Dies sind Faktoren, die auf die Bedürfnisse der Selbstverwirklichung und Wertschätzung zurückzuführen sind, also die Wachstumsbedürfnisse nach Maslow, oder die Motivatoren nach Herzberg. So ist es auch wichtig, dass Arbeitsleistungen, welche z.B. umfangreich, kompliziert oder dringend waren, auch geschätzt werden. Passiert dies nicht, kann das zur Demotivation führen, da der Mitarbeiter dann anfängt, seine Arbeit nicht mehr als wichtig zu erachten und infolgedessen seine Anstrengungsbereitschaft nachlässt. Auch und besonders bei Mitarbeitern, dessen Aufgaben reine Routine sind, ist es wichtig, diesen in gewissen Abständen klar zu machen, wie wichtig ihre Arbeit dennoch ist. Des Weiteren sollte man dem Mitarbeiter ein regelmäßiges Feedback zukommen lassen, damit dieser erkennt, dass sein Fleiß und seine Zuverlässigkeit von Anderen wahrgenommen und wertgeschätzt wird. Dabei gilt es vor allem, ehrlich zu sein. Empfindet er dies als reine Routine, wird kein motivierender Effekt zu erwarten sein, eher eine Abnahme der Motivation.

Geld liefert lediglich eine zeitlich begrenzte Motivation. Dies kann sicherlich auch jeder an sich selbst erkennen. Bekommt man eine Gehaltserhöhung, ist man zunächst einmal erfreut und motiviert, da man eine Bestätigung für die Qualität und das Engagement seiner Arbeit erhalten hat. Doch bereits nach ein paar Monaten wird das erhöhte Gehalt wieder als Normalität empfunden und verliert somit seine motivierende Wirkung. Wenn man Geld als Motivationsmittel benutzt, besteht sogar die Gefahr, dass sich die Motivation in Demotivation umschlägt, da die Mitarbeiter durch Gehalterhöhungen oder Prämien ebenso enttäuscht werden können, wenn sie dachten, diese fallen besser oder umfangreicher aus. Zudem besteht die allgemeine Gefahr, dass die Angestellten bei zu vielen finanziellen Anreizen, das eigentliches Interesse an ihren Arbeitsinhalten verlieren können, und somit auch ihre intrinsische Motivation. Auch Leistungszuschläge sind bei Mitarbeitern mit mehreren Aufgabenbereichen kritisch zu sehen. Es könnte

\footnotetext{
${ }^{55}$ Vgl. Laufer, Hartmut: Praxis erfolgreicher Mitarbeitermotivation S. $47 \mathrm{ff}$.
} 
passieren, dass sie die Aufgaben, bei denen sie keine Zulagen bekommen, vernachlässigen. Jedoch ist das Gehalt eine Grundvorrausetzung, dass Motivation überhaupt entstehen kann, was sich anhand der Bedürfnistheorie widerspiegelt. Es ist laut Maslow ein Defizitbedürfnis, und führt bei nicht vorhanden sein dazu, dass die Person unzufrieden ist, was einen Mangel an Motivation nach sich zieht. ${ }^{56}$

Auch die Unternehmen müssen einen Kulturwandel, von einer Ressourcenausnutzungskultur, hin zur einer Potenzialentfaltungskultur vollziehen. Ein besonderes Beispiel hierfür ist z.B. der dm-drogerie markt. Er zeigt auf, wie ein Unternehmen funktionieren sollte, damit die Mitarbeiter ihre Potenziale entfalten können. So werden die Lehrlinge schon mit Beginn ihrer ersten Arbeitstage gefordert. Sie bekommen von Anfang an Aufgaben zugetragen, welche sie eigenständig bewältigen müssen. Sind die Aufgaben schwieriger, soll der Lehrling zuerst Lösungswege für die Aufgabe erarbeiten, die er dann mit seinem Ausbilder bespricht. Bei dessen Zustimmung setzt er diese dann um.

Das Prinzip was dahinter steckt, ist das, dass der Lehrling sich intensiver mit seiner Arbeit auseinander setzt, da er keine Lösungswege vorgegeben bekommt, sondern diese selbst erarbeiten soll. Zusätzlich bleiben diese besser im Gedächtnis haften und sind später besser abrufbar. Die Ausbilder achten darauf, dass die übertragenen Arbeiten nicht zu einfach, aber auch nicht zu schwer zu bewältigen sind. Das Selbstvertrauen der Lehrlinge wächst dadurch nach und nach.

Als Höhepunkt der Ausbildung gehen die Ausbilder in andere Filialen des Unternehmens und überlassen vier Wochen lang die Leitung der Filiale den Nachwuchskräften. Auch an zwei Theaterworkshops, welche in den ersten zwei Jahren der Ausbildung stattfinden, nehmen die Lehrlinge teil und haben dabei erneut alles selbst in der Hand. Es liegt allein in ihren Händen, was sie machen und wie sie es machen. Damit wird erneut das Selbstbewusstsein der Auszubildenden gestärkt und die Zusammenarbeit gefördert. $^{57}$

Anhand dieses Beispiels des dm-drogerie marktes, lässt sich erkennen, dass die obersten Ebenen der Bedürfnistheorie nach Maslow angesprochen werden. Den Lehrligen wird von Beginn an die Verantwortung übertragen. Gleichzeitig können sie sich durch die Erstellung eigener Lösungswege selbst verwirklichen, haben große Handlungsspielräume und bauen eine enge Bindung zum Unternehmen auf. Durch die Bearbeitung der obersten Bedürfnisse sind sie gleichzeitig auch motiviert. Gerald Hüther

\footnotetext{
${ }^{56}$ Vgl. Laufer, Harthmut: Praxis erfolgreicher Mitarbeitermotivation S. $56 \mathrm{ff}$.

${ }^{57} \mathrm{Vgl}$. Kulturwandel in Unternehmen \& Organisationen, online: [URL] http://www.kulturwandel.org/content/die-unternehmen-des-gelingens/dm-drogerie-markt/, Stand 25.05.2015
} 
unterstützt diesen Kulturwandel in Unternehmen, indem er sagt "Wir brauchen Manager, die mit Leidenschaft führen und ihre Mitarbeiter begeistern. Eine der wichtigsten Erkenntnisse der Hirnforschung ist, dass Menschen nur dann ihre Potenziale entfalten, wenn sie sich für etwas begeistern. “58

${ }^{58}$ Hüther, Gerald: Lufthansa Exclusive Interview, online: [URL] http://www.geraldhuether.de/populaer/veroeffentlichungen-von-gerald-huether/zeitschriften/lufthansa-exclusive/index.php, Stand 20.05.2015 


\section{Fragebogen}

\subsection{Aufbau des Fragebogens}

Ziel des Fragebogens war es herauszufinden, inwiefern die Mitarbeiter des Fallbeispiels motiviert sind, bzw. woran es liegt, wenn dies nicht der Fall ist. Dadurch sollten Vergleiche zu den zuvor dargelegten Motivationstheorien gezogen werden, um zu sehen, inwieweit diese mit der Praxis konform gehen.

Durch die sich anschließende Auswertung des Fragebogens, und die daraus resultierenden Erkenntnisse über den Motivationsstand des Fallbeispiels, soll im selben Atemzug diesem eine Handlungsempfehlung gegeben werden, wie die Mitarbeiter zu diesem Zeitpunkt am besten motiviert werden können, bzw. welche Maßnahmen als erstes ergriffen werden müssten, damit diese in Zukunft motivierter sind.

Damit ein Vergleich zu den theoretischen Vorüberlegungen gezogen werden kann, sind die Fragen spezifisch gestellt und auf die Inhalte der Theorien angepasst.

Anhand der gestellten Fragen, soll zu einem die Gesamtsituation im Unternehmen widergespiegelt, und zum anderen eine Erkenntnis gewonnen werden, welche die häufigsten Bedürfnisse sind, die die Mitarbeiter befriedigen wollen. Nur dann können dem Unternehmen konkrete Handlungsempfehlungen, zur Verbesserung der Gesamtsituation gegeben werden.

Nach der Einleitung des Fragebogens, in der noch einmal der Sachverhalt geschildert wird, folgen zunächst einige einfache Einstiegsfragen, um die Teilnehmer an den Fragebogen heranzuführen. Die daran anschließenden sind bereits auf die Theorien abgestimmte Fragen, welche sich z.B. auf die Bedürfnistheorie, die Zwei-FaktorenTheorie oder die Zielsetzungstheorie beziehen.

Die Auswertung des Fragebogens und die daraus resultierenden Handlungsempfehlungen sind aufgrund der Betrachtung eines einzelnen Unternehmens nicht so repräsentativ, wie es bei anderen Fragebögen zu anderen allgemeinen Themen der Fall ist. Denn je nach Unternehmen, Branche oder auch Ort der Befragung, können die Bedürfnisse ganz unterschiedlich sein.

Es ist jedoch bei dieser Thematik auch schwierig eine repräsentative Befragung durchzuführen, da hierfür selbst bei Beschränkung auf den Raum Sachsen, zahlreiche Unternehmen hätten befragt werden müssen. Das hätte einerseits den Rahmen gesprengt und wäre andererseits im Rahmen dieser Arbeit nicht umsetzbar gewesen. 


\subsection{Auswahl des Fallbeispiels}

Das Fallbeispiel des Fragebogens ist die atlantics $\mathrm{GmbH}$, ein mittelständisches und inhabergeführtes Manufakturunternehmen, welches sich auf die Herstellung von Edelstahlrutschen spezialisiert hat. Der Autor tätigte im Rahmen seines Studiums, und auch schon davor, mehrere Praktika in diesem, knapp 40 Mitarbeiter umfassenden Unternehmen.

Da das Unternehmen dem Autor in Aussicht stellte, inn nach der Beendigung seines Studiums zu übernehmen, bot es sich an, die Befragung in dem Unternehmen durchzuführen. Er wollte damit herauszufinden, wie der Stand der Mitarbeitermotivation zum jetzigen Zeitpunkt ist. Gleichzeitig sollte herausgefunden werden, ob etwas verbessert werden kann bzw. muss und was genau das ist, um dies später möglicherweise auch selbst mit umzusetzen.

Es gibt in dem Unternehmen keine bestimmte Person, die für die Mitarbeiterführung ausgebildet ist, im Sinne einer Personalleitung. Das ist dem Grund geschuldet, dass es sich bei der vorliegenden Firma um eine kleine handelt, und die Personalleitungsaufgaben von dem Geschäftsführer sowie den zwei Projektleitern übernommen werden.

Der Fragebogen wurde im Zeitraum vom 20.05.2015 bis 27.05.2015 in der Firma ausgelegt und von den Mitarbeitern ausgefüllt. Da in diesem Zeitraum einige Mitarbeiter Urlaub hatten oder krank waren, konnte der Fragebogen nicht von allen Mitarbeitern ausgefüllt werden. Zum besseren Verständnis der folgenden Auswertung des Fragebogens, der daraus resultierenden Erkenntnisse, und darauf abgestimmten Handlungsempfehlungen, folgt eine kurze Erläuterung der Struktur des Unternehmens.

Die atlantics $\mathrm{GmbH}$ teilt sich in eine Büroabteilung und die Produktion auf, wobei der überwiegende Teil der Mitarbeiter in der Produktion tätig ist. Da es ein Manufakturunternehmen, also ein produzierendes Gewerbe ist, bilden Männer den Hauptbestandteil des Unternehmens. Die meisten Mitarbeiter, besonders in der Produktion, sind auch schon Familienväter. 


\subsection{Auswertung des Fragebogens}

Die Auswertung der Fragebögen für das Fallbeispiel, haben dem Autor interessante Ergebnisse über die Gesamtsituation im Unternehmen und der Mitarbeiter geliefert.
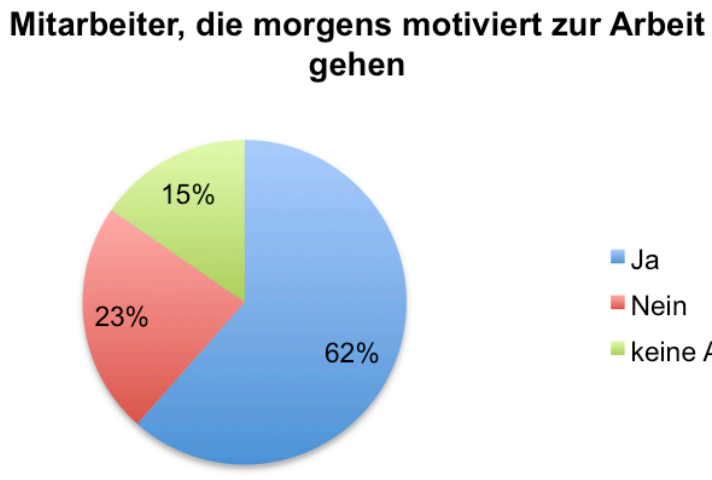

\section{Verbundenheitsgefühl mit dem} Unternehmen

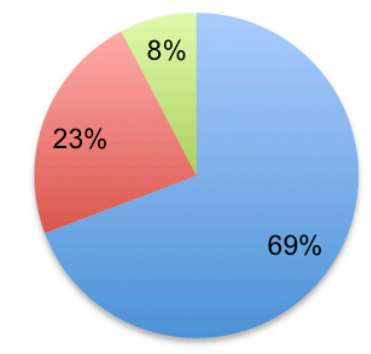

Abbildung 6: Auswertung der Fragen 1 \& 2

Mit den ersten beiden Fragen wollte der Autor in Erfahrung bringen, wie viele Mitarbeiter morgens motiviert zur Arbeit gehen und wie viele sich mit dem Unternehmen verbunden fühlen. Die Auswertung hat ergeben, dass über die Hälfte der Mitarbeiter motiviert zur Arbeit geht, und sich sogar noch mehr mit dem Unternehmen verbunden fühlen. Dieses Ergebnis zeigt, dass hier schon eine gute Basis vorhanden ist, denn immerhin gehen schon einmal über die Hälfte der Mitarbeiter motiviert zur Arbeit. Man kann auch erkennen, dass es dem Unternehmen möglich wäre, mehr motivierte Mitarbeiter zu haben, da sich mehr Mitarbeiter mit dem Unternehmen verbunden fühlen, als derzeit motiviert zur Arbeit gehen. Dies bietet eine gute Vorraussetzung, dass sich die Anzahl der motivierten Mitarbeiter möglicherweise in der Zukunft noch erhöhen könnte, denn Verbundenheit schafft Motivation. 
Fehltage der Mitarbeiter im vergangenem Jahr, aufgrund von Unwohlseins oder Krankheit

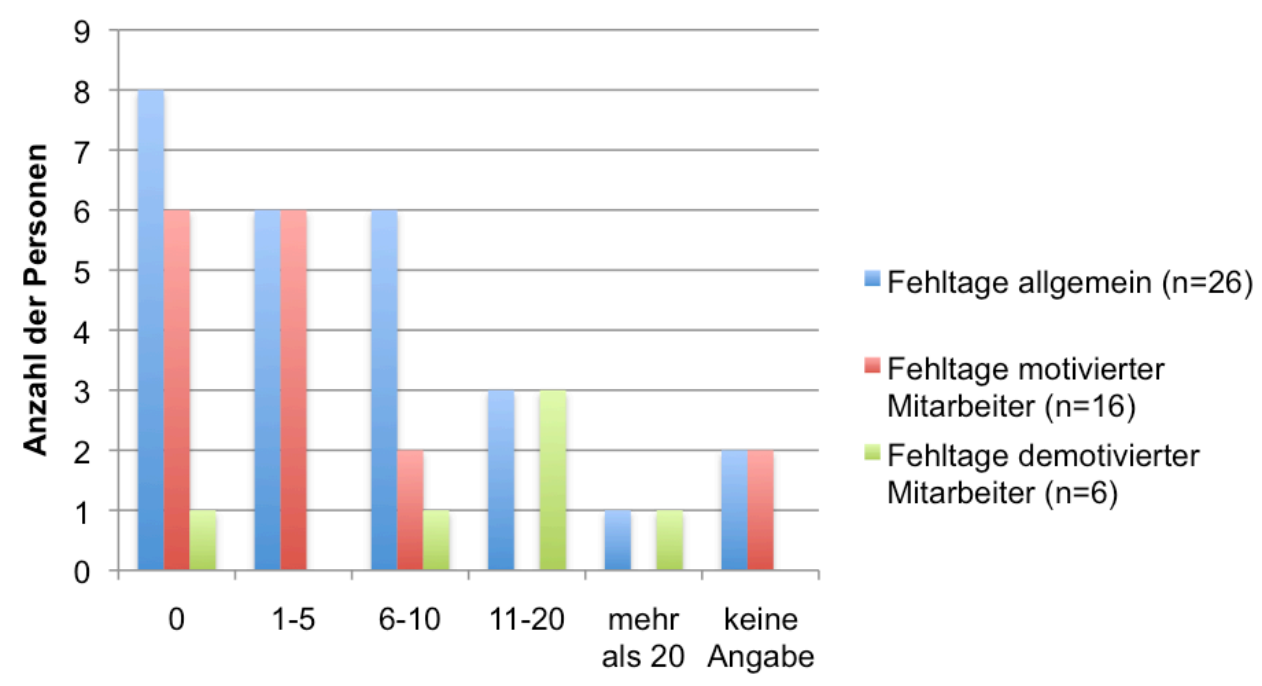

Anzahl der Fehltage

Abbildung 7: Auswertung der Frage 3

Mit der dritten Frage sollten die Fehltage der Mitarbeiter im vergangenem Jahr, auf Grund von Unwohlseins oder Krankheit ermittelt werden. Diese Frage wurde mit dem Hintergrund erstellt, herauszufinden, ob motivierte Mitarbeiter weniger Fehltage aufweisen als Demotivierte. Im Allgemeinen verzeichneten die Mitarbeiter im vergangenen Jahr 6-10 Fehltage. Diejenigen, die morgens motiviert zur Arbeit gehen, haben im Durchschnitt 1-5 Fehltage, während Mitarbeiter, die unmotiviert gehen im Durchschnitt 11-20 Fehltage aufweisen. Demnach ist hier ein deutlicher Unterschied zwischen motivierten und demotivierten Mitarbeitern erkennbar.

Die vierte Frage zielte darauf ab herauszustellen, ob die Mitarbeiter bei der Auflistung mehrerer Bedürfnisse, welche aus jeweils 6 Motivatoren und 6 Stabilisatoren bestanden, die Motivatoren an den ersten Stellen wählen, da diese als motivierend für den Mitarbeiter gelten. Die Stabilisatoren hingegen sollten befriedigt sein und sich damit an den hinteren Stellen wiederfinden, da die Nichtbefriedigung dieser Bedürfnisse, wie bereit erwähnt, zur Unzufriedenheit führt. Anhand der nachfolgenden Tabelle lässt sich jedoch erkennen, dass keine klare Trennung zwischen Motivatoren und Stabilisatoren gezogen werden kann. Dies zeigt weiterhin, dass an den Grundbedingungen im Unternehmen gearbeitet werden muss. Jedoch sollte man nicht darauf schließen, dass die Motivation in diesem Fall nur durch eine bessere Bezahlung gewährleistet werden kann. So kann das Verlangen nach einer besseren Bezahlung auch daher rühren, dass andere Bedürfnisse wie Anerkennung, ein gutes Betriebsklima, oder eine Wertschätzung der Arbeit nicht gewährleistet ist und die Mitarbeiter die Bezahlung daher als Entschädigung für diese Tatsachen ansehen. Oder die Begründung liegt in der Tatsache, 
dass alles immer teurer wird und die Menschen auf das Geld angewiesen sind, um sich private Bedürfnisse erfüllen zu können. Das wiederum hat eine motivierende Wirkung. Im nächsten Kapitel der Handlungsempfehlungen, wird noch einmal genauer Bezug darauf genommen.

\begin{tabular}{|l|l|}
\hline Motivationsfaktoren & Rangfolge \\
\hline eine gute Bezahlung meiner Arbeit, ein möglichst hohes Einkommen (Stabilisator) & 1 \\
\hline Anerkennung guter Arbeitsergebnisse durch meine Vorgesetzten (Motivator) & 2 \\
\hline Eigenverantwortliches Arbeiten, persönliche Entscheidungsbefugnisse (Motivator) & 3 \\
\hline interessante Arbeitsinhalte sowie herausfordernde Arbeitsaufgaben (Motivator) & 4 \\
\hline gute zwischenmenschliche Beziehungen im Unternehmen (Stabilisator) & 5 \\
\hline eine humane Führungskultur im Unternehmen (Stabilisator) & 6 \\
\hline die Sicherheit des Arbeitsplatzes, eine gute soziale Absicherung (Stabilisator) & 7 \\
\hline $\begin{array}{l}\text { Gestaltungsmöglichkeiten und Handlungsfreiheiten innerhalb des Aufgabenberei- } \\
\text { ches (Motivator) }\end{array}$ & 8 \\
\hline Arbeitsaufgaben, die erkennbare Erfolgserlebnisse ermöglichen (Motivator) & 9 \\
\hline eine gute Position im Unternehmen (Stabilisator) & 10 \\
\hline Aufstiegsmöglichkeiten bei persönlicher Bewährung (Motivator) & 11 \\
\hline eine gute Arbeitsplatzausstattung (Stabilisator) & 12 \\
\hline
\end{tabular}

Tabelle 1: Auswertung der Frage 4

Diese Erkenntnis spiegelt sich auch in der Auswertung der Frage 5 und 6 wieder. Hier ist erkennbar, dass das Betriebsklima, welches alle Mitarbeiter als wichtig erachten, im Durchschnitt jedoch nur als befriedigend empfunden wird. Daher findet sich das Betriebsklima bei Frage 4 auch an fünfter Stelle wieder. Daraus lässt sich schließen, dass Mitarbeiter bei Verbesserung des Betriebsklimas, automatisch viel motivierter wären. Dies könnte infolgedessen dazu führen, dass mehr Mitarbeiter morgens motiviert zur Arbeit gehen und sich auch mit dem Unternehmen verbunden fühlen.

\section{Beurteilung des Betriebsklimas}

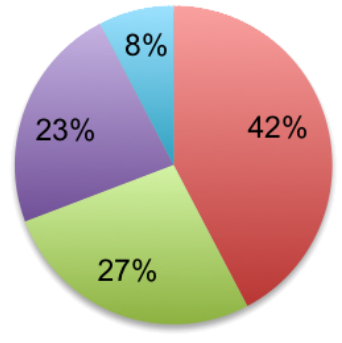

\section{Betriebsklima wird als wichtig erachtet}

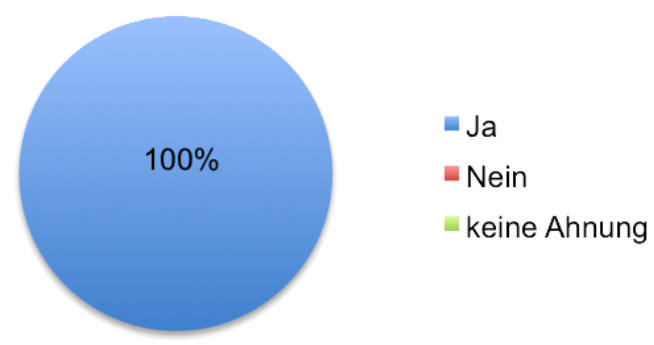


Die Auswertung der Frage 7 ist ebenfalls positiv ausgefallen. Der Durchschnitt der Mitarbeiter kommuniziert täglich mit dem Vorgesetzten. Dies stellt erneut eine gute Grundlage für die folgenden Handlungsempfehlungen dar, da es wichtig ist, dass die Führungskraft grundsätzlich oft mit den Angestellten kommuniziert.

\section{Häufigkeit der Kommunikation mit dem Vorgesetzten}

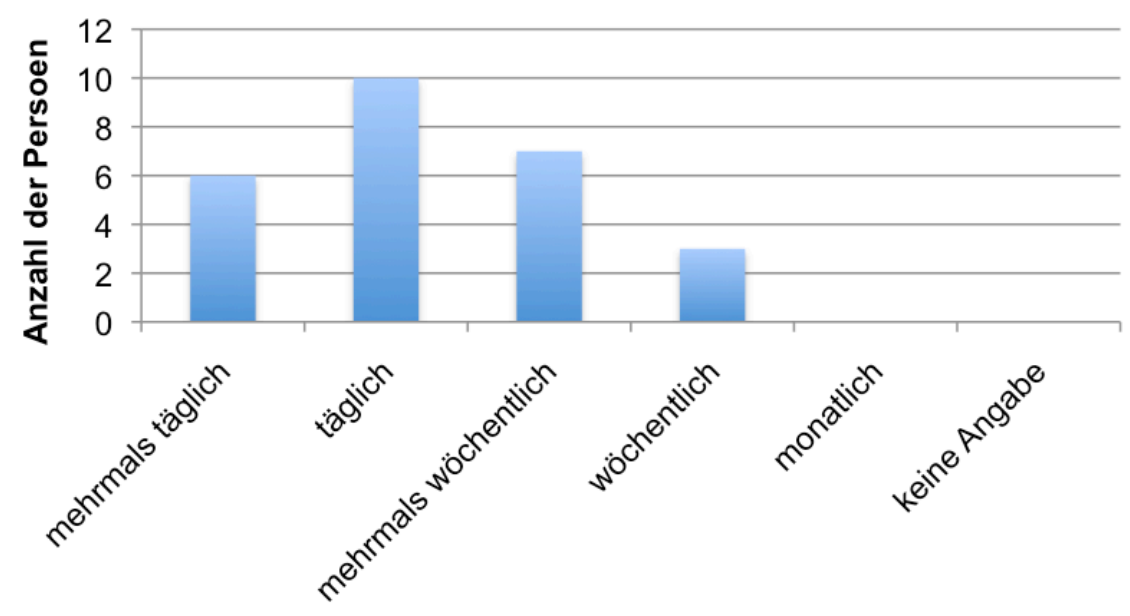

Abbildung 9: Auswertung der Frage 7

Ein ebenfalls positives Ergebnis liefert die Auswertung der Frage 8, in der die Mitarbeiter angaben, dass sie die Ausstattung ihres Arbeitsplatzes im Durchschnitt als gut empfinden. Dies ist wichtig, da eine gute Ausstattung des Arbeitsplatzes eine Grundvoraussetzung ist. Das zeigt sich auch in Frage 4, da hier die Ausstattung des Arbeitsplatzes auf den letzten Platz gewählt wurde und somit am wenigsten motivierend auf die Mitarbeiter wirkt, da dieser schon als gut empfunden wird.

\section{Beurteilung der Arbeitsplatzausstattung}

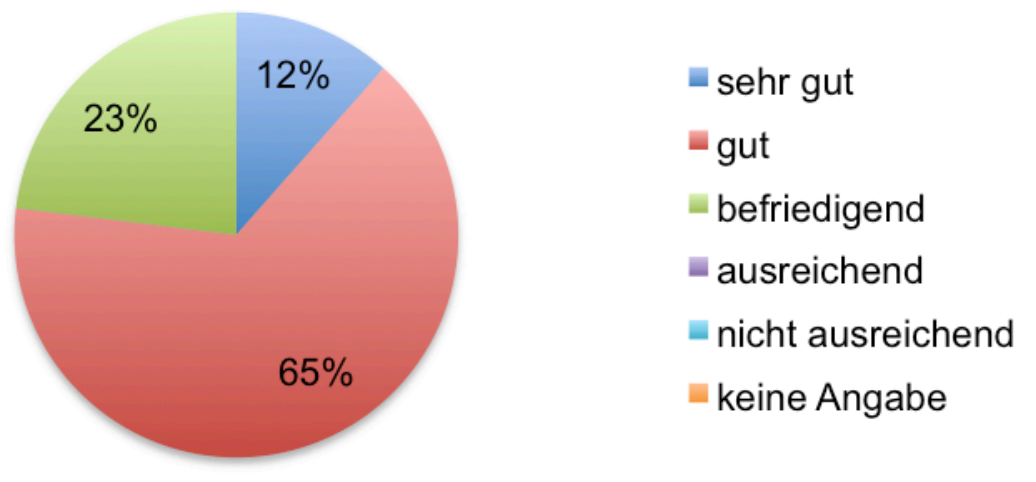


Mit den Antworten der Frage 9 und 10 wollte der Autor in Erfahrung bringen, ob die Mitarbeiter oft Überstunden leisten oder nicht, und ob dies für sie ein Problem darstellt. Die Auswertung der Fragen hat gezeigt, dass etwas mehr als die Hälfte der Befragten Überstunden leisten. Die knappe Hälfte gab zudem an, dass Überstunden für sie ein Problem darstelle.

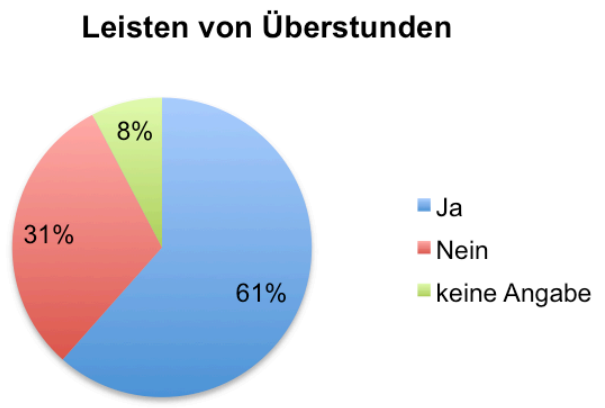

Überstunden als Problemfaktor

Abbildung 11: Auswertung der Fragen 9 \& 10

Durch die Beantwortung der Frage 11 lies sich erkennen, dass bei einem kleinen Teil der Mitarbeiter das Privatleben unter der Arbeit leidet. Die genauere Auswertung hat ergeben, dass diese Personen alle Überstunden leisten und fast alle von innen zudem angaben, dass Überstunden ein Problem für sie darstelle. Da die Überstunden zumeist nicht mehrere Tage vorher angekündigt werden können, sondern erst wenige Tage vorher angeordnet werden, steht die Vermutung nahe, dass in diesem Fall auch Mitarbeiter betroffen sind, welche Kinder haben und durch die kurzfristige Ankündigung von Überstunden Probleme bekommen. Möglich wäre auch, dass es sich hier um Mitarbeiter handelt, welche anderweitigen Verpflichtungen nachkommen müssen. Dies wurde bereits in der Auswertung der Fragen 9 \& 10 erläutert. Das stellt eine wichtige Erkenntnis für die sich anschließende Handlungsempfehlung dar.

\section{Privatleben leidet unter der Arbeit}

\section{Überstunden als Problemfaktor}

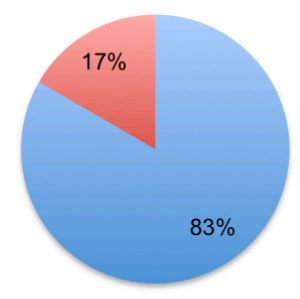

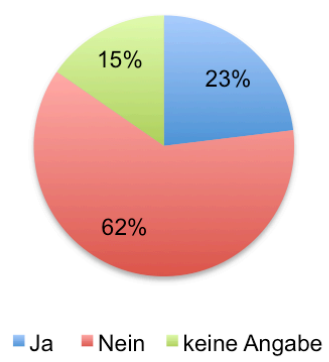

$=\mathrm{Ja}=$ Nein $\quad$ keine Angabe
Leisten von Überstunden

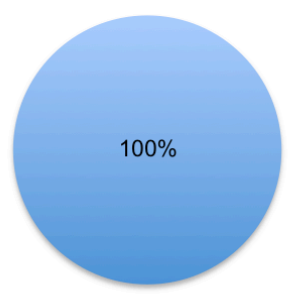


Zudem hat sich herausgestellt, dass sich die Mehrheit der Mitarbeiter nicht angemessen bezahlt fühlt. Nach genauerer Betrachtung stelle sich heraus, dass diese Mitarbeiter nicht eigenverantwortlich handeln können, kein regelmäßiges Feedback erhalten, oft Überstunden leisten und ihre erbrachten Leistungen nicht anerkannt werden. Diese Faktoren können unter anderem dafür sorgen, dass der Mitarbeiter sich auch trotz eines angemessenen Gehaltes, hinsichtlich seiner Position, unterbezahlt fühlt.

\section{Bezahlung ist angemessen}

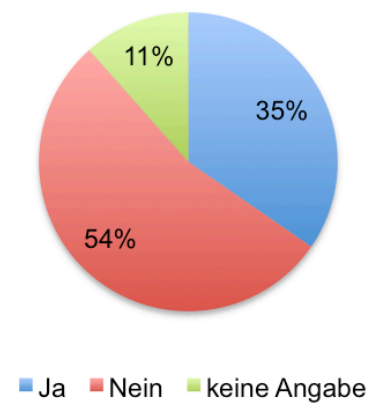

Befugnis eigenverantwortlich zu handeln

\section{Leisten von Überstunden}

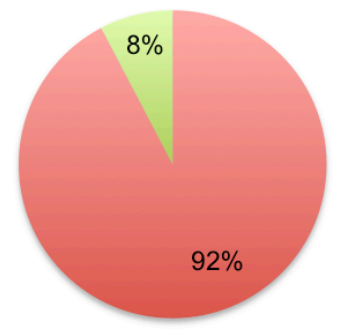

Erhalt eines regelmäßigen Feedbacks

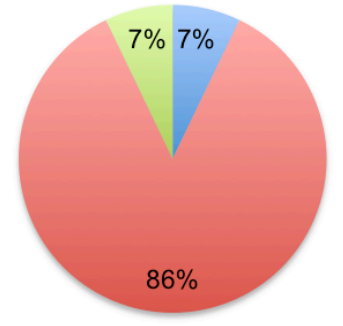

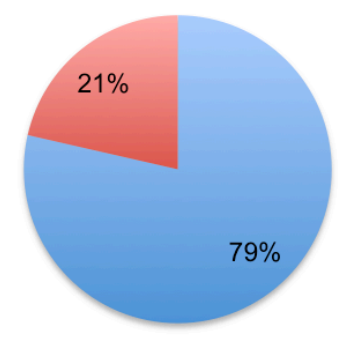

Anerkennung erbrachter Leistungen

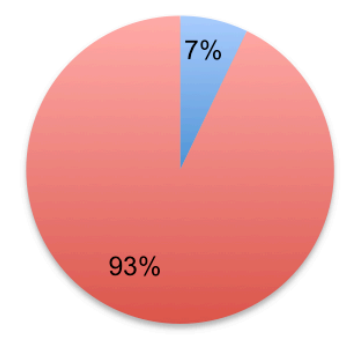

Abbildung 13: Auswertung der Frage 12 
Positiv ist zu sehen, dass die Mehrheit der Mitarbeiter angab, in Entscheidungs(findungs)prozesse mit einbezogen zu werden. Dies ist eine wichtige Vorraussetzung, da hiermit dem Mitarbeiter vermittelt wird, dass jede einzelne Meinung auch wichtig und relevant ist.

\section{Mitwirkung bzw. Anteilnahme an Entscheidungs(findungs)prozessen}

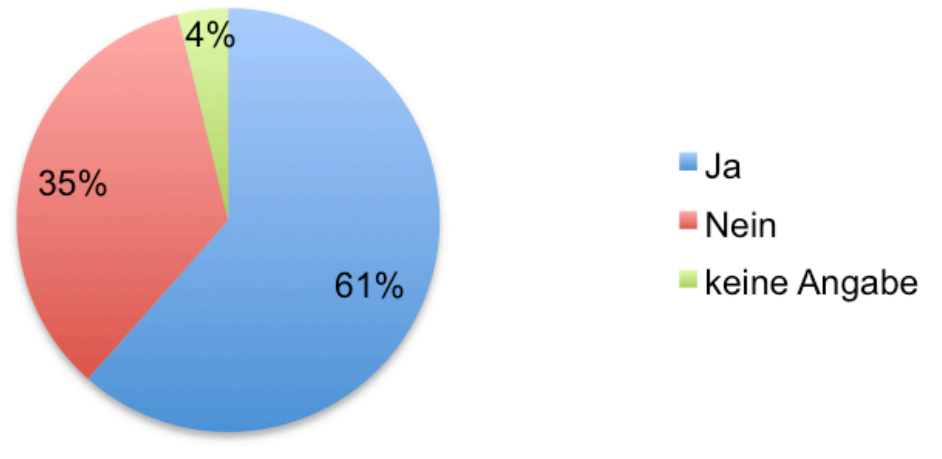

Abbildung 14: Auswertung der Frage 13

An der Tatsache, dass kaum jemand ein regelmäßiges Feedback erhält, sollte man unbedingt arbeiten, da es sehr wichtig für die Mitarbeiter, und auch deren Motivation ist.

\section{Erhalt eines regelmäßigen Feedbacks}

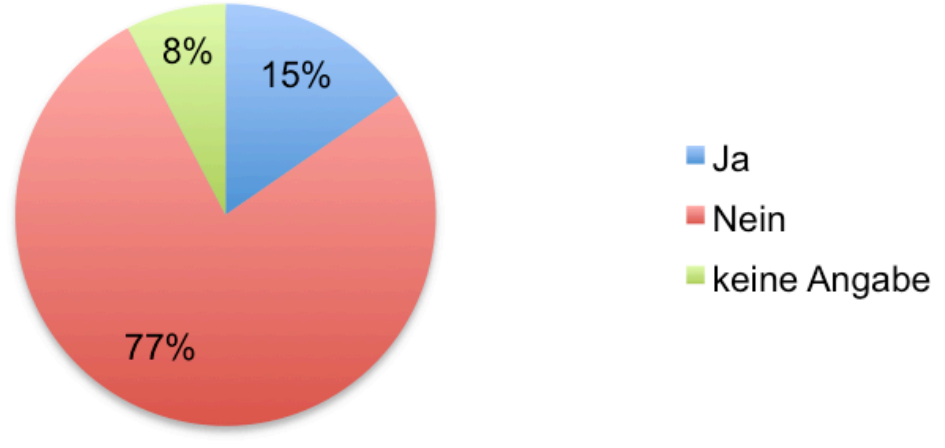

Abbildung 15: Auswertung der Frage 14 
Bei Aufgabe 15 stellte sich heraus, dass die Mehrheit der Mitarbeiter ihre erbrachten Leistungen nicht anerkannt bekommen. Mitarbeiter, deren Leistungen anerkannt werden, fühlen sich im Ergebnis auch angemessen bezahlt. Fehlt diese jedoch, fühlen sich die Angestellten zumeist auch nicht gut bezahlt. Aus diesem Grund ist es wichtig, jedem ein Gefühl von Anerkennung seiner Arbeit entgegen zu bringen.

\section{Anerkennung erbrachter Leistungen}

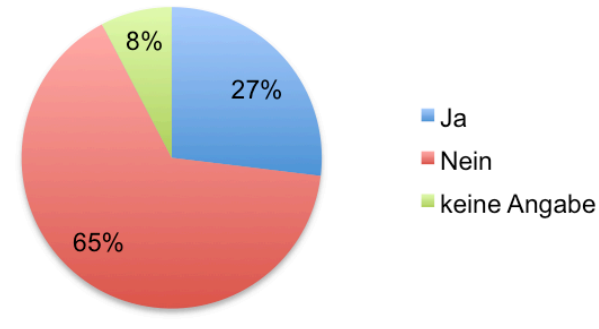

\section{Anerkennung erbrachter Leistungen} im Verhältnis zur Bezahlung

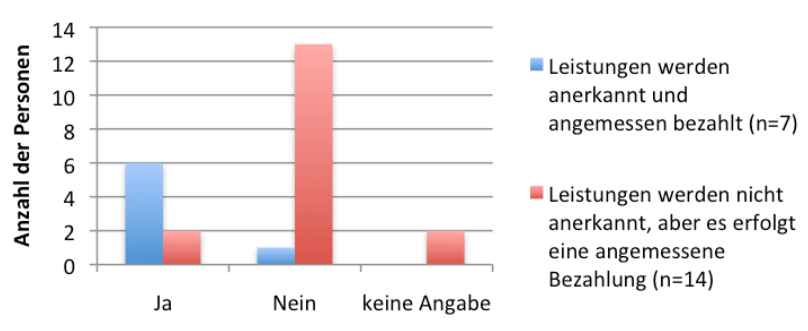

Abbildung 16: Auswertung der Frage 15

Die Auswertung der Frage 16 hat aufgezeigt, dass nur wenige Mitarbeiter befugt sind, eigenverantwortlich zu Handeln. Es ist also zu prüfen, welche Mitarbeiter im Unternehmen eigenverantwortlich handeln können bzw. welchen Mitarbeitern man mehr Eigenverantwortung zutragen könnte. Denn dies wirkt sich förderlich auf die Motivation aus.

\section{Befugnis eigenverantwortlich zu handeln}

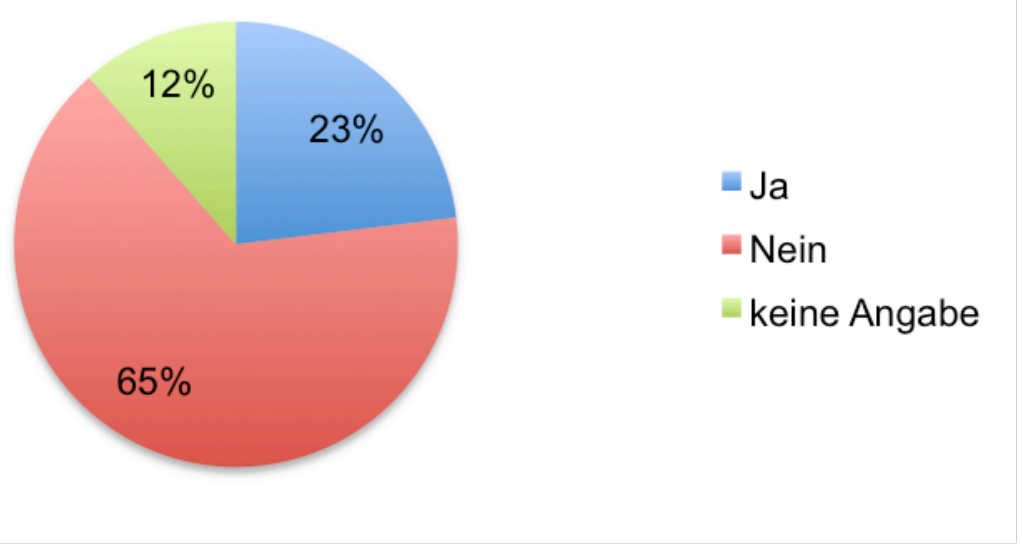


Mit der Frage 17 wollte der Autor herausfinden, wie routiniert die Arbeit der einzelnen Mitarbeiter ist. Die Auswertung ergab, dass der Durchschnitt der Mitarbeiter eher weniger routinierten Aufgaben nachgeht. Wichtig ist jedoch, dass es auch eine gewisse Anzahl an Mitarbeitern gibt, dessen Arbeitsaufgaben routiniert sind - eine weitere wichtige Erkenntnis für die spätere Handlungsempfehlung.

\section{Routinegrad der Tätigkeits- und Aufgabenfelder}

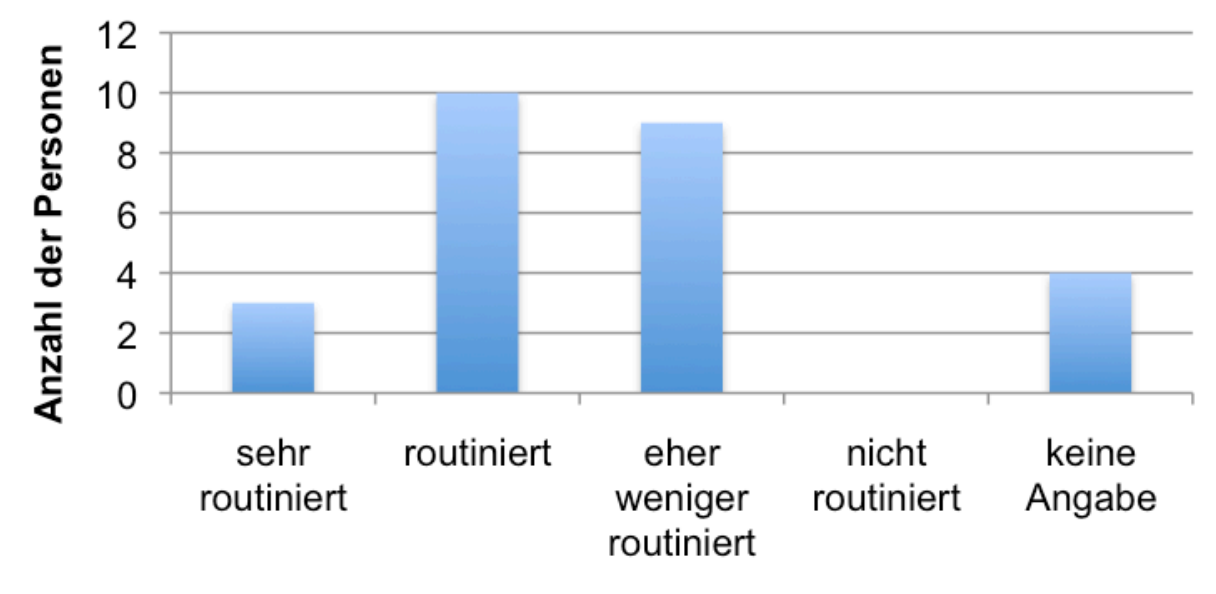

Abbildung 18: Auswertung der Frage 17

In der Frage 18 sollten die Mitarbeiter angeben, ob sie präzise formulierte Arbeitsaufträge erhalten, da dies auch ein Faktor ist, der zur Motivation des Mitarbeiters beiträgt, und das Resultat das Ergebnis wesentlich beeinflussen kann. Anhand der Auswertung zeigte sich, dass es sich die Waage hält. Das könnte mit der subjektiven Meinung des Einzelnen zutun haben, oder aber es ist abhängig davon, ob man im Büro oder Produktion tätig ist.

\section{Erhalt von präzise formulierten Arbeitsaufträgen}

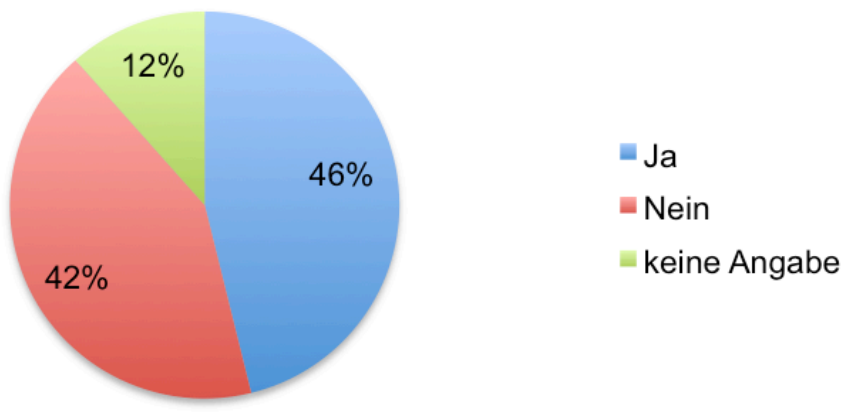


In der letzten Frage wollte der Autor in Erfahrung bringen, was sich der Mitarbeiter wünschen würde, wenn er einen Wusch frei hätte. Hier fand er heraus, dass sich die meisten Mitarbeiter eine Gehaltserhöhung wünschen, gefolgt von mehr Urlaubstagen, oder flexibleren Arbeitszeiten. Die genaue Betrachtung zeigt, wie es auch die Theorie sagt, dass Mitarbeiter, welche mit ihrem Gehalt zufrieden sind, sich eher mehr Urlaubstage, mehr Anerkennung oder flexiblere Arbeitszeiten wünschen, und keine Gehaltserhöhung. Diejenigen, welche sich nicht angemessen bezahlt fühlen, wünschen sich infolgedessen zumeist eine Gehaltserhöhung, was auch die nachfolgende Abbildung noch einmal verdeutlicht.

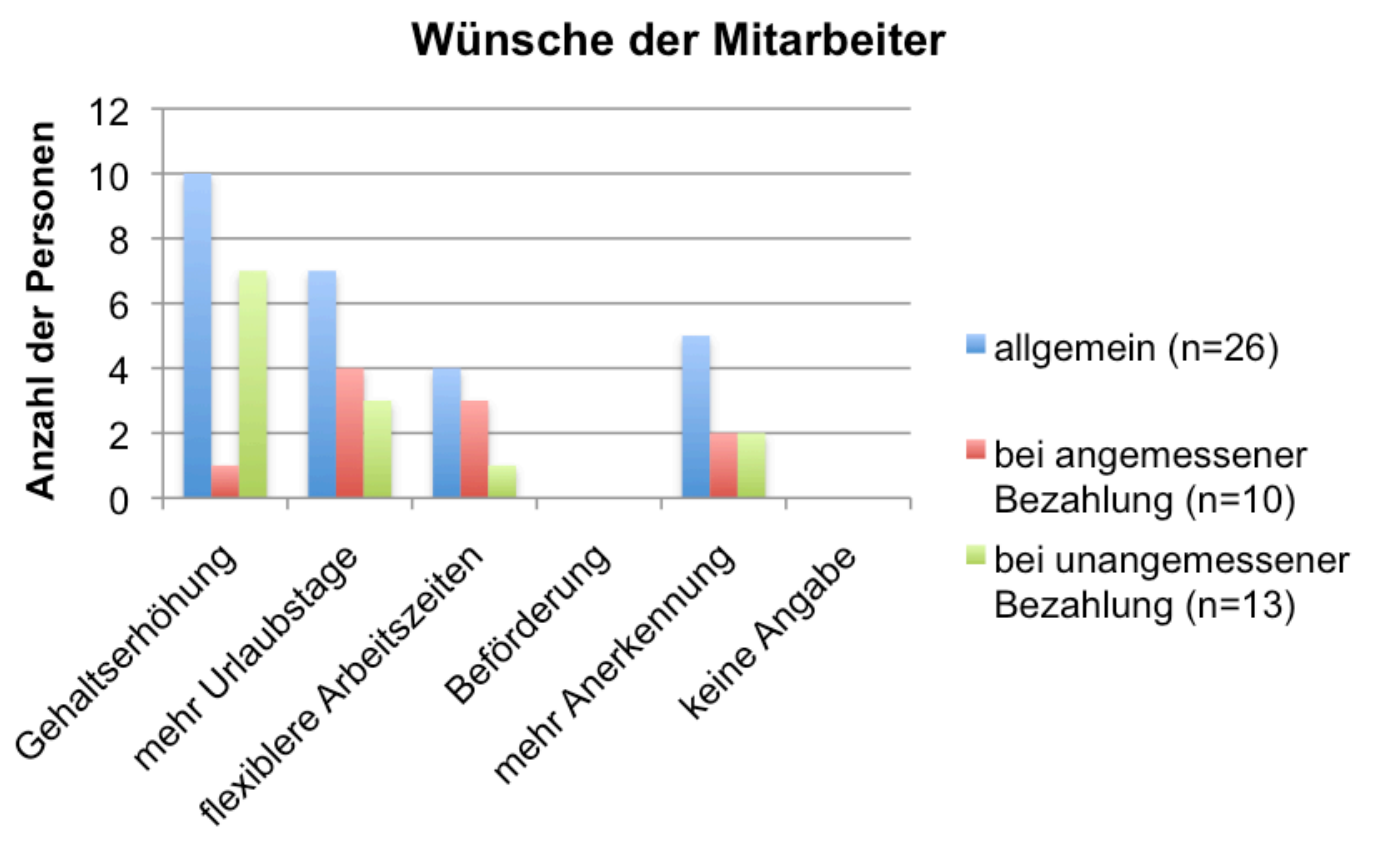

Abbildung 20: Auswertung der Frage 19

\subsection{Handlungsempfehlung}

Mittels der Auswertung des Fragebogens, und der daraus gewonnenen Erkenntnisse über die Mitarbeiter und den Zustand im Unternehmen, wird nun im Folgenden eine Handlungsempfehlung erläutert. Dabei bietet das Unternehmen bereits gute Bedingungen, da immerhin schon mehr als die Hälfte aller befragten Mitarbeiter motiviert sind und sich mit dem Unternehmen verbunden fühlen. Dennoch ist die Anzahl der motivierten Mitarbeiter noch ausbaufähig, da es für ein Unternehmen wichtig ist, motivierte Angestellte zu haben. Nicht nur dahingehend dass diese, wie bereits erwähnt, Ideen in das Unternehmen einbringen und mehr Leistungen hervorrufen können, sondern auch um Fehltage zu verringern, oder durch Demotivation entstehenden Krankheiten vorzu- 
beugen, was dem Unternehmen auch Kosten verursacht. Demzufolge ist lediglich etwas mehr als die Hälfte noch zu wenig.

Das Erste was das Unternehmen tun sollte, ist an seinem Betriebsklima zu arbeiten. Die Mitarbeiter sind der Meinung, dass dieses lediglich befriedigend in der Firma zu sehen ist und das obwohl alle Mitarbeiter das Betriebsklima als so wichtig empfinden, und es sogar auf Platz 5 der motivierenden Faktoren wählten. Wenn das Betriebsklima verbessert werden würde, würden möglicherweise auch die Mitarbeiter ein Stück weit motivierter zur Arbeit kommen und sich vielleicht auch noch mehr mit dem Unternehmen verbunden fühlen. Wer geht schon gern zur Arbeit, wenn kein Gemeinschaftsgefühl bzw. Akzeptanz unter den Kollegen herrscht. Es macht mehr Spaß, in einem Unternehmen zu arbeiten, in dem alle Mitarbeiter miteinander auskommen, da sie sich so auch gegenseitig motivieren können.

Hierfür sollte der Arbeitgeber, oder die Führungspersonen im Unternehmen herausfinden, was der Grund für das befriedigende Betriebsklima ist. Tragen dafür eine oder mehrere Personen/Angestellte die Verantwortung, oder sogar die Führungsebene selbst. Der Vorteil hierbei ist, dass das Betriebsklima zu verbessern keine Kosten mit sich bringt, wie z.B. eine Gehaltserhöhung der Mitarbeiter, und trotzdem zur Motivation beiträgt.

Des Weiteren sollte das Unternehmen bzw. die Führungspersonen daran arbeiten, den Mitarbeitern für erbrachte Leistungen Anerkennung zu schenken, und innen auch mehr Feedbacks zu den erbrachten Leistungen zu geben bzw. während der Bearbeitung des Ziels. Dabei sollte darauf geachtet werden, dass bei einer Leistung, die nicht der Vorstellung der Führungsebene entspricht, im Feedback eine Kritik erfolgt, bei der sich der Mitarbeiter nicht gedemütigt, sondern zu einer Verbesserung angehalten fühlt. Auch negative Kritik ist positiv zu sehen, da sie dazu führt, dass sich gemachte Fehler nicht wiederholen, wenn man freundlich darauf aufmerksam gemacht wurde und dadurch auch keine Resignation erfolgt. Es ist ebenso gut, wenn ein Feedback während der Bearbeitung der Aufgabe erfolgt, da der Mitarbeiter durch dieses z.B. mitbekommt, ob er noch im zeitlichen Rahmen liegt, der Vorgesetzte mit der bisherigen Arbeit zufrieden ist, oder noch Änderungen vorgenommen werden müssen, und der Arbeitsprozess nicht erst nach Beendigung eventuell noch einmal komplett wiederholt werden muss. Durch das „rechtzeitige“ Feedback können möglicherweise auch Überstunden oder umfangreiche Nacharbeitungen verhindert werden. Gutes sollte ebenfalls angemerkt werden, und im Falle von der atlantics $\mathrm{GmbH}$ auch, was später aus den geplanten und gefertigten Produkten geworden ist, sowie gegebenenfalls die Kundenzufriedenheit an den jeweiligen Angestellten weitergeleitet werden. 
Da der Großteil der Mitarbeiter des Unternehmens angab, dass ihre Aufgaben routiniert seien, ist es ebenso wichtig, den betroffenen Personen zu verdeutlichen, dass die Aufgabe zwar routiniert ist und wenig Vielfalt sowie Herausforderungen bietet, aber das sie dennoch von höchster Wichtigkeit für das Endergebnis ist. Das wiederum vermittelt innen, dass Ihre Aufgabe genau so wichtig ist, wie die der Kollegen.

Die Kommunikation ist demnach ein wichtiger Faktor, welcher im Unternehmen jedoch schon recht gut ausgeprägt ist. Die Mitarbeiter kommunizieren im Durchschnitt täglich mit ihrem Vorgesetzten, was eine gute Vorraussetzung für die folgenden Punkte darstellt.

Bei der Frage nach den Überstunden stellte sich in der Auswertung heraus, dass Überstunden zu leisten, für die meisten Mitarbeiter ein Problem darstellt. Bei der Frage, ob das Privatleben unter der Arbeit leidet, bestätigten dies 6 Personen, welche alle Überstunden leisteten. 5 von Ihnen kreuzten an, dass Überstunden für sie ein Problem darstellen. Hierfür ist es wichtig herauszufinden, warum Überstunden ein Problem darstellen. Dies könnten z.B. die Mitarbeiter sein, die Kinder und infolgedessen weniger Zeit für sie haben, oder bestimmten Verpflichtungen dadurch nachkommen müssen. Es könnte aber auch daran liegen, dass Überstunden zumeist kurzfristig angekündigt werden und dies eventuelle Terminplanungen der Mitarbeiter durcheinander bringt. In diesem Fall wird dem Unternehmen oder der Führungsperson empfohlen, dafür zu sorgen, dass erforderliche Überstunden so früh wie möglich angemeldet werden, damit sich die betroffenen Mitarbeiter darauf einstellen können, oder gegebenenfalls ein anderer Mitarbeiter die Überstunden übernehmen könnte und sich derjenige später einmal revanchiert. Außerdem könnte man unter den Angestellten fragen, wer bereit wäre diese zu vollziehen, da es zumindest einige Mitarbeiter gab, denen Überstunden nichts ausmachen.

Des Weiteren ist es auch wichtig, dass die Führungsperson bei der Vergabe von Arbeitsaufgaben dafür sorgt, dass sich die Mitarbeiter mit ihrer Aufgabe und dem gesetzten Ziel identifizieren können und das diese auch präziser formuliert werden. Hierfür wird der Führungsperson empfohlen, die Arbeitsaufträge so zu formulieren, dass der Mitarbeiter diese auch wirklich versteht und inm alle wichtigen Rahmendaten bekannt sind. Nur wenn dieser sein Ziel zu 100\% verstanden und verinnerlicht hat, wird er die inm übertrage Aufgabe zur vollsten Zufriedenheit erfüllen können. Zudem wäre zu empfehlen, sich bei der Vergabe der Arbeitsaufträge mit dem Mitarbeiter zu unterhalten, um herauszufinden, ob dieser sich zukünftig eine etwas schwierigeren Aufgabe zutrauen würde, oder sogar den Wunsch danach verspürt mehr gefördert zu werden und neue Herausforderungen anzutreten. 
Das Ergebnis aus der Auswertung der Frage 16, in der nur knapp ein Viertel des Personals angab eigenverantwortlich handeln zu dürfen, war für den Autor nicht unerwartet. Dies ist dennoch als kritisch zu erachten, da die Mitarbeiter durch die fehlende Eigenverantwortung demotiviert werden, und infolgedessen auch die Bindung zum Unternehmen stark nachlässt. Dies löst ein Misstrauensgefühl aus, was dafür sorgt, dass die Mitarbeiter das Gefühl vermittelt bekommen, dass Ihnen selbständiges Handeln nicht zugetraut wird. Daher ist es wichtig, seine Mitarbeiter mehr selbstständig arbeiten zu lassen. Hier könnte sich das Unternehmen ein Beispiel an dem dmdrogerie markt nehmen, und dessen Unternehmenskultur, welche bereits im Kapitel: „Wie können Mitarbeiter motiviert werden?“, erläutert wurde. Dass dies auch Erfolge bringt, zeigen mehrere Beispiele, die wiederum auch mehrere Möglichkeiten aufzeigen, wie man Mitarbeiter dazu bringt eigenverantwortlich zu handeln.

Viele Mitarbeiter gaben an, für ihre Arbeit nicht angemessen bezahlt zu werden. Angemessen ist zwar ein subjektives Empfinden, aber dennoch bleibt die Tatsache bestehen, dass sich die Mehrheit der Mitarbeiter unterbezahlt fühlt. Anhand der vorgestellten Motivationstheorien kann dieses Empfinden mehrere Ursachen haben. Es könnte, wie in der ERG-Theorie nach Alderfer beschrieben, an den Folgen, ein bestimmtes Bedürfnis nicht befriedigen zu können, was infolgedessen ein niedrigeres Bedürfnis zu befriedigen verstärkt, liegen. Auch die nur zum Teil gegebenen Stabilisatoren nach Herzberg können bei dem Mitarbeiter einen Zustand auslösen, dass dieser Unzufriedenheit verspürt, welches er in der Annahme, dieses durch ein höheres Gehalt zu mindern versucht. Es kann aber auch einfach daran liegen, das wie bereits in der Valenz-Instrumentalitäts-Erwartungstheorie erwähnt, der Mitarbeiter ein hohes Gehalt zu diesem Zeitpunkt am motivierendsten empfindet, um z.B. sein Haus fertig zu bauen oder sich einen höheren Lebensstandard erfüllen zu können. Dieser ist heute auch weitaus höher angesiedelt, als es noch vor einigen Jahren der Fall war. Es könnte aber auch sein, dass das Gehalt wirklich zu niedrig ist. In diesem Fall ist eine Gehaltserhöhung von Nöten, um dieses Bedürfnis befriedigen zu können. Die Erkenntnisse, welche in der Theorie schon vorweg gewonnen wurden, spiegelten sich auch in der Auswertung der Frage 4 und 12 in den Fragebögen wieder.

Es lässt sich erkennen, dass die Gründe, warum sich ein Mitarbeiter nicht angemessen bezahlt fühlt, unterschiedliche Ursachen haben können. Alle diese soeben genannten Ursachen, lassen verschiedene Optionen ableiten, wie die Führungsperson die Mitarbeiter motivieren kann.

Der Führungsperson wird empfohlen, durch Gespräche mit jedem einzelnen Mitarbeiter herauszufinden, was der Grund jedes Einzelnen dafür ist, dass dieser sich nicht angemessen bezahlt fühlt. Hierfür benötigt dieser Zeit, sich intensiv mit den einzelnen Mitarbeitern auseinander zu setzen. Er muss eine Bindung zu seinen Mitarbeitern 
aufbauen, damit diese wissen, dass sie ihm auch alles anvertrauen können und die Führungsperson ihnen auch ernsthaft zuhört, und sich für die Belange der Mitarbeiter interessiert. Sobald die Mitarbeiter das Gefühl haben, dass die Führungsperson ehrlich an innen interessiert ist, ein offenes Ohr hat, wird er schnell herausfinden, was der Grund jedes einzelnen Mitarbeiters ist. Zudem sorgt das auch erneut dafür, dass das Betriebsklima und der Zusammenhalt im Unternehmen verbessert wird, was dafür sorgen kann, dass die Mitarbeiter auch bereit sind für das Unternehmen oder die Führungsperson einzuspringen, wenn einmal Not am Mann ist. Durch die offene Kommunikation mit den Mitarbeitern werden inm des Weiteren auch andere Sachen aufgezeigt, wo Verbesserungen getätigt werden könnten.

Sollten zu diesem Zeitpunkt schon die vorangegangenen Handlungsempfehlungen umgesetzt sein, kann sich das Verlangen der Mitarbeiter nach einem höheren Gehalt bis dahin schon gelegt haben. Nach dem Motivationsfaktor Gehalt, kamen nämlich laut der Auswertung der Frage 4, die Faktoren der Wertschätzung und der Selbstverwirklichung. Diese gehören laut Maslow zu den Wachstumsbedürfnissen, oder nach Herzberg, zu den Motivatoren, und führe dazu, dass der Mitarbeiter zufrieden ist und infolgedessen auch motiviert. Daher wird dem Unternehmen ebenso empfohlen, die Handlungsempfehlungen der Reihenfolge nach zu realisieren und zwischendurch nach jedem Schritt zu schauen, wie die Situation jetzt ist, bzw. was noch verändert werden muss. Um die Wirkung zu überprüfen wäre auch ein erneuter Fragebogen nach einer gewissen Zeit eine Möglichkeit. Damit kann überprüft werden, ob sich tatsächlich etwas verändert hat und die Mitarbeiter jetzt motivierter und zufriedener sind. 


\section{Resümee}

\subsection{Zusammenfassung und Reflektion der Ergebnisse}

Die Frage, wer für die Motivation der Mitarbeiter verantwortlich ist, lässt sich anhand der gewonnenen Erkenntnisse der Arbeit damit beantworten, dass sowohl die Unternehmen, als auch der Arbeitgeber oder unmittelbare Vorgesetzte, für die Motivation der Mitarbeiter verantwortlich sind. Diese haben es in der Hand, die Bedingungen für motivierte Mitarbeiter zu schaffen. Wer Mitarbeiter als Ressource ansieht, die es auszunutzen gilt, anstatt zu versuchen, die Potenziale jedes Einzelnen auszuschöpfen, wird es nie schaffen, diese zu motivieren. Demzufolge wird er auch nie die volle Leistung der Mitarbeiter erfahren. Mitarbeitern muss mehr Vertrauen geschenkt werden, damit diese auch eigenverantwortlich handeln können. Ihre Arbeit muss anerkannt und wertgeschätzt werden. Grundlegende Bedingungen, wie ein gutes Betriebsklima, eine gute Arbeitsplatzausstattung, ein angemessenes Gehalt oder auch eine gute und ehrliche Beziehung zu dem Vorgesetzten sollten ermöglicht werden, sodass diese wissen, dass das Unternehmen auch an innen interessiert ist und es zu schätzen weiß, sie als Angestellte zu haben. Gleichzeitig sollten dann auch die Arbeitnehmer ihre Vertragbedingungen zur vollsten Zufriedenheit des Arbeitgebers ausführen, bzw. ihr bestmögliches geben, damit ein Geben und Nehmen entsteht.

In der Befragung im Unternehmen atlantics kam zwar heraus, dass die Mitarbeiter das Gehalt als den motivierendsten Faktor ansahen, jedoch gleichzeitig waren $100 \%$ der Befragten der Ansicht, dass das Betriebsklima sehr wichtig ist. Es ist darauf zu schließen, dass dies also auch ein Faktor ist, der sie sehr motiviert. Selbstverständlich ist Geld ein wichtiger Aspekt. Alles wird stetig teurer und man benötigt das Geld, um sich einen guten Lebensstandard zu schaffen - denn das ist unter anderem ein Grund, wofür man arbeiten geht. Aber eine „Hochbezahlung“, wie es auch Rüdiger Hossiep verlauten lies, motiviert einen Menschen ebenso wenig.

Vielmehr sollte das Gehalt angemessen sein. Bei einem solchen Gehalt, inklusive Faktoren wie Anerkennung, Wertschätzung und der Gleichen, wird der Angestellte viel motivierter sein, als durch eine höchstmögliche Bezahlung. Motivation ist vorrangig auch eine Sache der Seele und des Gefühls. Ein angemessenes Gehalt muss kein hohes, vielmehr ein gerechtes Gehalt sein. Ein Anderer sollte für die gleiche Arbeit nicht deutlich mehr Lohn bekommen. Es ist aber auch einfach eine Tatsache, dass ein Kleinunternehmen nicht so viel zahlen kann, wie es einem Großkonzern vielleicht möglich ist. Selbst wenn dies gewollt wäre. Unter dieser Maßgabe würde das der Mitarbeiter vielleicht sogar verstehen und die anderen Faktoren, die das Unternehmen zur 
Motivation schafft, mehr schätzen. Das ganze funktioniert aber nur, wenn es dem Angestellten auch kommuniziert wird.

Der Grund dafür, dass Mitarbeiter demotiviert sind, liegt meistens daran, dass die soeben genannten Bedingungen nicht erfüllt sind. Dies zeigte sich auch anhand der Auswertung der Fragebögen. Die Bedingungen müssen gegeben sein, um die intrinsischen Motivation zu erwecken, sodass die Mitarbeiter ihre Arbeit mit Freude und Bereitschaft verrichten, sowie Ideen oder Verbesserungen in das Unternehmen einbringen und nicht nur Dienst nach Vorschrift leisten.

Es hat sich zudem gezeigt, dass die Mitarbeiter nicht anhand fixer Motivationsstrategien motiviert werden können. Dies erfordert vielmehr ein persönliches Verhältnis und ehrliches Interesse der Führungsperson an dem Mitarbeiter, um herauszufinden, welche Bedürfnisse dieser ganz speziell zu befriedigen versucht und ihm dahingehend Anreize zu liefern, die zur Befriedigung dieser beitragen. Daher kann man auch nicht verallgemeinern, wie ein Mitarbeiter genau zu motivieren ist, da jeder Mitarbeiter, je nach seinen persönlichen Umständen, an einem anderen Punkt steht und andere Bedürfnisse zu befriedigen versucht.

Die Arbeit hat dem Autor aufgezeigt, wie Mitarbeiter durch den Arbeitgeber oder den Vorgesetzten motiviert werden können, was häufige gemachte Fehler sind, und wie diese vermieden werden können. Es lies auch erkennen, dass Mitarbeiter nicht anhand festgelegter Strategien motiviert werden können. Zudem wurde anhand eines reellen Beispieles weiterhin aufgezeigt, wie wichtige Erkenntnisse über den Zustand der Mitarbeiter eines Unternehmens ermittelt werden können, um daraus Handlungsempfehlungen ableiten zu können.

Kurz gesagt setzt das Unternehmen, inklusive Führungsebene selbst, die Grundlage dafür, motivierte Mitarbeiter zu schaffen. Denn nur wer es schafft, seine Mitarbeiter zu motivieren, wird zukünftig konkurrenzfähig bleiben und die Mitarbeiter an das Unternehmen binden. Nicht zu vergessen ist dabei - das Herzstück eines jeden Unternehmens sind seine Angestellten. 


\section{Literaturverzeichnis}

DAK-Gesundheit: DAK-Gesundheitsreport 2015. URL:

http://www.dak.de/dak/download/Vollstaendiger bundesweiter Gesundheitsreport 201 5-1585948.pdf, Stand 22.05.2015

Deutsche Akademie für Management: Zielsetzungstheorie (Locke/Latham). URL: https://www.akademie-management.de/fernstudium-management/glossarmanagementtheorien/zielsetzungstheorie, Stand 15.05.2015

Duden Online: Motivation. URL: http://www.duden.de/rechtschreibung/Motivation, Stand 08.05.2015

Gabler Wirtschaftlexikon: Motivation. URL: http://wirtschaftslexikon.gabler.de/Definition/motivation.html, Stand 08.05.2015

Gabler Wirtschaftslexikon: intrinsische Motivation. URL:

http://wirtschaftslexikon.gabler.de/Definition/intrinsische-motivation.html, Stand 08.05.2015

Göstl, Caterina: Mitarbeitermotivation: Erkenntnisse aus der psychologischen Forschung erfolgreich in der Wirtschaft anwenden. - Saarbrücken: VDM Verlag, 2010

Haarhaus, Benjamin: Herzbergs Zwei-Faktoren-Theorie. URL:

http://arbeitszufriedenheit.net/herzbergs-zwei-faktoren-theorie/, Stand 13.05.2015

Hurrelmann, Klaus; Klotz, Theodor; Haisch, Jochen: Lehrbuch für Prävention und Gesundheitsförderung. - 4. Aufl. - Bern: Verlag Hans Huber, 2014

Hüther, Gerald (2015): Lufthansa Exclusive Interview. URL: http://www.geraldhuether.de/populaer/veroeffentlichungen-von-gerald-huether/zeitschriften/lufthansaexclusive/index.php, Stand 20.05.2015

Hüther, Gerald; Purps-Pardigol, Sebastian: Kulturwandel in Unternehmen \& Organisationen. URL: http://www.kulturwandel.org/content/die-herausforderung/, Stand 08.05.2015

Hüther, Gerald; Purps-Pardigol, Sebastian: Kulturwandel in Unternehmen \& Organisationen: DM-DROGERIE MARKT. URL: http://www.kulturwandel.org/content/dieunternehmen-des-gelingens/dm-drogerie-markt/, Stand 25.05.2015 
Katrin Matyssek, Anne: Führung und Gesundheit: Ein praktischer Ratgeber zur Förderung der psychosozialen Gesundheit im Betrieb: - 3. Aufl. - Norderstedt: Books on Demand $\mathrm{GmbH}, 2012$

Laufer, Hartmut: Praxis erfolgreicher Mitarbeitermotivation: Techniken, Instrumente, Arbeitshilfen. - Offenbach: GABAL Verlag, 2013

Motzkuhn, Mario: Wie geht Motivation wirklich?: Betriebliche Anreizsysteme auf dem Prüfstand: Gängige Anreizpraktiken von Unternehmen im Licht der modernen Motivations- und Gehirnforschung. - Hamburg: Diplomica Verlag, 2013

Nink, Marco: Engagement Index Deutschland 2014. URL: http://montua-partner.de/wpcontent/uploads/2015/04/Präsentation-zum-Engagement-Index-2014.pdf, Stand 05.05.2015

Stock-Homburg, Ruth: Personalmanagement: Theorien, Konzepte, Instrumente. - 2. Aufl. - Wiesbaden: Gabler Verlag, 2010

Thunig, Christian: Interview mit Reinhard K. Sprenger. URL:

http://www.karriere.de/unternehmen/ein-unternehmen-das-eine-suchanzeigeaufgeben-muss-hat-schon-verloren-166108/2l, Stand 20.05.2015

www.zitate.de: Motivation. URL: http://www.zitate.de/kategorie/Motivation, Stand 05.05.2015

www.zitate.de: Motivation. URL: http://www.zitate.de/kategorie/Motivation?page=5, Stand 06.05.2015 


\section{Anlagen}

HOCHSCHULE

MITTWEIDA

UNIVERSITY OF

APPLIED SCIENCES

\section{Fragebogen zur Mitarbeitermotivation}

Sehr geehrte Damen und Herren,

ich bin Student der Hochschule Mittweida und führe im Rahmen meiner Bachelorarbeit eine Befragung zum Thema Mitarbeitermotivation durch. Ziel des Ganzen ist es herauszufinden, welche Faktoren in der heutigen Zeit einen hohen Stellenwert besitzen und welche eine untergeordnete Rolle bei der Motivation eines Mitarbeiters spielen. Mithilfe dieses Fragebogens möchte ich vergleichen, inwiefern theoretische Vorüberlegungen mit der Praxis übereinstimmen.

Die Bearbeitung des Fragebogens wird ca. 15 Minuten in Anspruch nehmen und erfolgt anonym.

Ausgefüllte Fragebögen werden zudem streng vertraulich behandelt und lediglich zur Auswertung bzw. daraus resultierenden Schlussfolgerungen verwendet.

Für Rückfragen stehe ich Ihnen gern unter der nachfolgenden E-Mail Adresse zur Verfügung.

nkrumbie@hs-mittweida.de

Vielen Dank für Ihre Unterstützung!

Mit freundlichen Grüßen

Nic Krumbiegel 
Frage 1: Gehen Sie morgens motiviert zur Arbeit?
$\square$ Ja
$\square$ Nein
$\square$ keine Angabe

Frage 2: Fühlen Sie sich mit Ihrem Unternehmen verbunden?

$\square$ Ja $\square$ Nein $\square$ keine Angabe

Frage 3: Wie viele Fehltage verzeichneten Sie in etwa im vergangenen Jahr, aufgrund von Unwohlseins oder Krankheit?

$\square 0$

$\square 11-20$ $\square 1-5$

mehr als 20
6-10

keine Angabe 
Frage 4: Geben Sie an, durch was Sie bei Ihrer Arbeit motiviert werden. Bringen Sie die Motivationsfaktoren nach Ihrer Wichtigkeit in eine Rangfolge. Tragen Sie hierfür von Faktor 1 (motiviert mich am meisten) bis 12 (motiviert mich am wenigsten) in die Tabelle ein. (keine Doppelnennungen).

\begin{tabular}{|c|c|c|}
\hline $\mathrm{Nr}$. & Motivationsfaktoren & Rangfolge \\
\hline 1 & Aufstiegsmöglichkeiten bei persönlicher Bewährung & \\
\hline 2 & eine gute Position im Unternehmen & \\
\hline 3 & interessante Arbeitsinhalte sowie herausfordernde Arbeitsaufgaben & \\
\hline 4 & eine humane Führungskultur im Unternehmen & \\
\hline 5 & eine gute Arbeitsplatzausstattung & \\
\hline 6 & gute zwischenmenschliche Beziehungen im Unternehmen & \\
\hline 7 & eine gute Bezahlung meiner Arbeit, ein möglichst hohes Einkommen & \\
\hline 8 & Anerkennung guter Arbeitsergebnisse durch meine Vorgesetzten & \\
\hline 9 & eigenverantwortliches Arbeiten, persönliche Entscheidungsbefugnisse & \\
\hline 10 & $\begin{array}{l}\text { Gestaltungsmöglichkeiten und Handlungsfreiheiten innerhalb des Auf- } \\
\text { gabenbereiches }\end{array}$ & \\
\hline 11 & Arbeitsaufgaben, die erkennbare Erfolgserlebnisse ermöglichen & \\
\hline 12 & die Sicherheit des Arbeitsplatzes, eine gute soziale Absicherung & \\
\hline
\end{tabular}


Frage 5: Wie beurteilen Sie das Betriebsklima?

$\begin{array}{lll}\square \text { sehr gut } & \square \text { gut } & \square \text { befriedigend } \\ \square \text { ausreichend } & \square \text { nicht ausreichend } & \square \text { keine Angabe }\end{array}$

Frage 6: Erachten Sie ein gutes Betriebsklima als wichtig?
$\square \mathrm{Ja}$
$\square$ Nein
keine Angabe

Frage 7: Wie oft kommunizieren Sie mit Ihrem Vorgesetzten?

$\square$ mehrmals täglich

$\square$ täglich

$\square$ monatlich

$\square$ mehrmals wöchentlich

$\square$ wöchentlich

$\square$ keine Angabe

Frage 8: Wie beurteilen Sie die Ausstattung Ihres Arbeitsplatzes?

$\square$ sehr gut

$\square$ ausreichend

$\square$ gut

$\square$ befriedigend

$\square$ nicht ausreichend

$\square$ keine Angabe

Frage 9: Leisten Sie oft Überstunden?

$\square \mathrm{Ja}$

Nein

keine Angabe

Frage 10: Stellen Überstunden für Sie ein Problem dar?

$\square$ Ja

$\square$ Nein

keine Angabe

Frage 11: Leidet Ihr Privatleben unter der Arbeit?

$\square \mathrm{Ja}$

Nein

keine Angabe

Frage 12: Werden Sie für Ihre Arbeit angemessen bezahlt?

$\mathrm{Ja}$

$\square$ Nein

keine Angabe 
Frage 13: Werden Sie in Entscheidungs(findungs)prozesse einbezogen?
$\square \mathrm{Ja}$
$\square$ Nein
keine Angabe

Frage 14: Erhalten Sie ein regelmäßiges Feedback bezüglich ihrer Arbeit?
$\square \mathrm{Ja}$
Nein
keine Angabe

Frage 15: Werden die von Ihnen erbrachten Leistungen anerkannt?
$\square \mathrm{Ja}$
$\square$ Nein
keine Angabe

Frage 16: Sind Sie befugt, eigenverantwortlich zu handeln?
$\square \mathrm{Ja}$
$\square$ Nein
keine Angabe

Frage 17: Wie routiniert sind die Tätigkeits- bzw. Aufgabenfelder Ihrer Arbeit?

$\square$ sehr routiniert

$\square$ routiniert

eher weniger routiniert

$\square$ nicht routiniert

$\square$ keine Angabe

Frage 18: Erhalten Sie präzise formulierte Arbeitsaufträge?

$\square \mathrm{Ja}$

Nein

keine Angabe

Frage 19: Was würden Sie sich am ehesten wünschen, wenn Sie einen Wunsch frei hätten? (keine Mehrfachnennung)

$\square$ Gehaltserhöhung

$\square$ Beförderung $\square$ mehr Urlaubstage

mehr Anerkennung flexiblere Arbeitszeiten

keine Angabe 


\section{Eigenständigkeitserklärung}

Hiermit erkläre ich, dass ich die vorliegende Arbeit selbstständig und nur unter Verwendung der angegebenen Literatur und Hilfsmittel angefertigt habe. Stellen, die wörtlich oder sinngemäß aus Quellen entnommen wurden, sind als solche kenntlich gemacht. Diese Arbeit wurde in gleicher oder ähnlicher Form noch keiner anderen Prüfungsbehörde vorgelegt.

Ort, Datum

Vorname Nachname 